\title{
Maximum resonance and cancellation phenomena in orthotropic plates traversed by moving loads: application to railway bridges
}

\author{
M.D. Martínez-Rodrigo ${ }^{\mathrm{a}, *}$, E. Moliner ${ }^{\mathrm{a}}$, A. Romero ${ }^{\mathrm{b}}$, G. De Roeck ${ }^{\mathrm{c}}$, P. Galvín ${ }^{\mathrm{b}}$ \\ ${ }^{a}$ Universitat Jaume I, Department of Mechanical Engineering and Construction, Avda. Sos Baynat s/n, ES-12071 \\ Castellón, Spain \\ ${ }^{b}$ Escuela Técnica Superior de Ingeniería, Universidad de Sevilla, Camino de los Descubrimientos s/n, ES-41092 Sevilla, \\ Spain \\ ${ }^{c}$ Department of Civil Engineering, K.U.Leuven, Kasteelpark Arenberg 40, B-3001 Leuven, Belgium
}

\begin{abstract}
The vibrational response of railway bridges is an issue of main concern, especially since the advent of High-Speed traffic. In the case of short-to-medium lengths and simply-supported spans excessive transverse acceleration levels may be induced at the platform, with detrimental consequences for passengers and infrastructures. The orthotropic plate has proven to be an appropriate model for the prediction of the response of certain typologies in the aforementioned cases such as multiple girder decks, solid or voided slabs or filler-beam multiple-track decks. In this contribution, the vibrational response of orthotropic plates, simply and elastically supported, circulated by vertical moving loads is investigated. First, maximum free vibration and cancellation conditions are derived analytically. From these, bridge span length-characteristic distance ratios leading to maximum and minimum resonances under series of equidistant loads are depicted. Second, the applicability of these ratios in oblique decks is analysed for the most common first three mode shapes: first longitudinal bending, first torsion and first transverse bending modes, and the errors in relation to the straight reference case are bounded. To this end, an extensive bridge catalogue of girder bridges is designed in the range of lengths of interest, covering flexural stiffnesses typical from both conventional and High-Speed railway lines. Finally, the applicability of the previous theoretical results is exemplified with experimental measurements performed on a bridge from the Spanish railway network.
\end{abstract}

Keywords: Railway bridges, orthotropic plates, resonance, cancellation, moving loads.

\section{Introduction}

Since the opening of the first High-Speed (HS) railway line in the 1960s in Japan, railway infrastructures have evolved dramatically in most developed countries. During the last decades, several HS lines have been constructed and many conventional lines have been upgraded to higher operating speeds. Spain is one of

\footnotetext{
* Corresponding author.

Email address: mrodrigo@uji.es (M.D. Martínez-Rodrigo)
} 
5 the leading countries in this regard with almost $2600 \mathrm{~km}$ of operational HS lines [1]. The increase of the design speeds brings along difficulties and new challenges. In particular, railway bridges must accomplish now strict requirements in terms of maximum vibration levels for the sake of passenger comfort and traffic safety. In this context, the dynamic behaviour of such structures has received considerable attention from the engineering and scientific community in the past decades.

This investigation focuses on the particular case of short-to-medium length $(10-25 \mathrm{~m})$ simply-supported (SS) bridges, which are prone to experience high transverse acceleration levels at the platform due to their usually associated low mass and structural damping [2, 3, 4, 5]. In countries like Spain in the aforementioned range of lengths common deck typologies built in the 80's and 90's for double track platforms are voided or solid concrete slabs, pseudo-slabs and precast decks composed by double-T beams [6] (see Fig. 1). In the last years, twin cell box girder decks are becoming more common due to their enhanced dynamic performance.

With the exception of the lastest, the vertical dynamic response of the aformentioned structures may be adequately predicted with orthotropic plate models, showing good correspondence with experimental measurements [7]. For this reason, the response of orthotropic plates under moving loads and its application to railway bridges constitutes the main interest of this work.

(a)

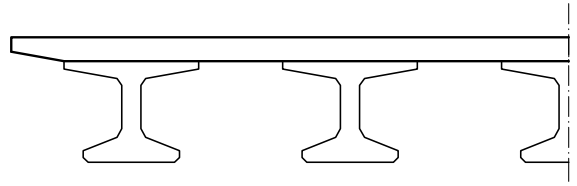

(c)

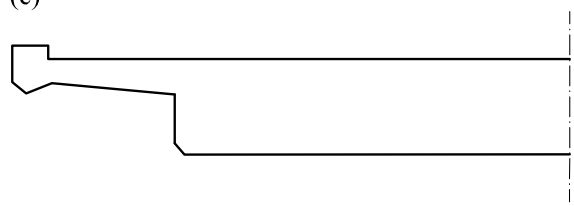

(b)

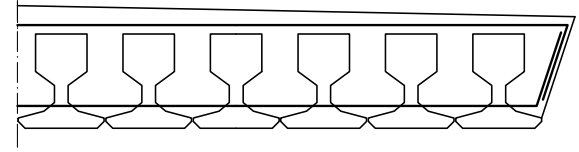

(d)

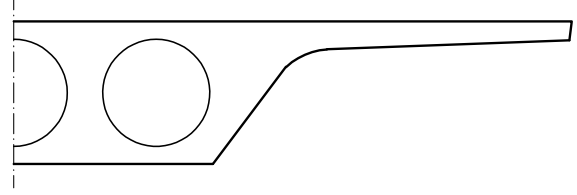

Figure 1: Railway bridge decks common for short-to-intermediate lengths: (a) precast double-T girder deck; (b) concrete pseudo-slab; (c) concrete solid slab; (d) concrete voided slab.

The basic phenomenon that governs the level of vibrations induced on a bridge by the circulation of a railway convoy is the amplitude of the free vibrations that a single axle leaves on the structure after its passage. Depending on the ratio between the travelling time of the load crossing the bridge and a certain natural period of the structure, the amplification of the free vibration response in that particular mode may be maximum or may be theoretically cancelled, implying that the structure would remain at rest under certain ideal conditions (i.e. in the absence of structural damping). This basic problem has been analysed in detail by authors such as Yang et al. 8, Savin [9] and Museros et al. [10] for SS beams and also for beams resting on vertical elastic supports (ES beams in what follows). The aim and novelty of this 
investigation is to extend this study and evaluate its validity to orthotropic plates representative of bridges with a dynamic behaviour characterized by modal contributions different from the longitudinal bending ones (i.e. first torsion, first transverse bending modes). This is the case of SS multiple track decks with length to width ratios close to unity and sections similar to those included in Figs. 1(a-d). In general, these structures exhibit several natural modes below $30 \mathrm{~Hz}$ which may significantly contribute to the maximum vertical acceleration [7].

The interest of knowing a priori the maximum free vibration and cancellation speeds for a certain structure is diverse. First, if a train induces resonance on a bridge, the dynamic amplification will depend on the closeness of the resonant velocity to these maximum free vibration or cancellation conditions. If the resonant speed coincides with a maximum free vibration condition, important amplification levels should be expected. On the contrary, if it takes place in the vicinity of a cancellation speed, the resonant peak will become imperceptible. Even though it is well known that in a real situation resonant amplitudes depend on 40 several factors, e.g. number of convoys, vehicle-structure and soil-structure interaction, and other damping and amplitude dependent mechanisms, the amplitude of the free vibrations can be understood as the basic phenomenon. Second, when amplitude dependent magnitudes must be determined from an experimental campaign performed on a bridge with a test vehicle, e.g. modal damping, the test speed should be selected such that it leads to a representative level of vibrations in the structure, avoiding close to cancellation 45 velocities.

The problem of transverse vibrations of beams and plates subjected to moving forces has received considerable attention in the past due to its applications in structural engineering. Nevertheless, the number of studies on plates is rather limited compared to those devoted to beams with different boundary conditions [11. Gbadeyan and Oni [12] formulated the dynamic problem of Rayleigh beams and plates under an arbitrary number of moving masses based on modified generalized finite integral transforms. Shadnam et al. 13] introduced a new method to compute the transient response of a rectangular plate excited by relatively large masses along arbitrary trajectories. Lee and Yhim [14 analysed single and two-span continuous composite plate structures subjected to multi-moving loads taking into account third order transverse shear deformation and rotary inertia. Au and Wang [15] investigated the vibratory response of rectangular orthotropic thin plates with general boundary conditions traversed by moving loads with the aim of determining the acoustic pressure distribution around the plate in the time domain. Gbadeyan and Dada [16] analysed the maximum internal forces and displacements of Mindlin elastic plates under uniform partially distributed moving masses. Malekzadeh et al. [17, 18, and Ghafoori et al.[19] investigated the dynamic response of composite plates under moving loads. Zhu and Law [20] analysed the dynamic behaviour of orthotropic plates simply supported on a pair of parallel edges applying Lagrange's equations and modal superposition. More recently some authors have investigated the resonance phenomena caused by the circulation of periodic forces or masses following either a semi-analytical [21, 22] or a numerical approach [23, 24, 7]. Neverthe- 
less, in the authors' opinion, regardless of the approach adopted, resonance phenomena caused by a series of periodic loads and the conditions for its maximization or cancellation depending on the free vibration amplitudes left by each single load have not been analysed in the case of orthotropic plates, especially when these separate from ideal well-known conditions i.e. SS boundary conditions and non-oblique configurations. This work constitutes a contribution in this area.

In what follows, first, the transverse vibration problem of a rectangular Kirchhoff orthotropic plate under the circulation of a single load crossing it at constant speed moving parallel to its longitudinal axis is formulated analytically, both for SS and ES boundary conditions on two opposite edges. Then, the nondimensional speeds for maximum free vibration and cancellation after the force leaves the structure are derived for a generic mode of vibration. Second, equating the maximum free vibration and cancellation speeds to resonant conditions induced by trains of equidistant loads, $L / d_{k}$ ratios for maximized resonances and cancelled resonances are derived, where $L$ stands for the bridge span length and $d_{k}$ for the characteristic distance of the train causing resonance. Third, an extensive catalogue of representative girder bridges is pre-designed covering lengths between 10 and $25 \mathrm{~m}$, bending flexural stiffnesses representative of both conventional and HS lines bridges, four levels of obliquity and two different support conditions, including and neglecting the vertical flexibility of the neoprene bearings at the supports. The applicability of the cancellation, maximum free vibration and, indirectly, $L / d_{k}$ values for maximized or cancelled resonances derived analytically is evaluated for the 112 bridges of the catalogue and the errors are bounded for the first three vibration modes, showing a good correspondence even in the case of structures that differ considerably from the reference straight plate. Finally, the applicability of the conclusions is depicted through the experimental response measured on a real HS railway bridge undergoing resonance.

\section{Free vibrations of orthotropic rectangular plates under a single moving load}

The partial differential equation governing the transverse oscillations $w(x, y, t)$ of a Kirchhoff orthotropic rectangular plate under a generic load distribution $q_{z}(x, y, t)$ (Fig. 2a) neglecting shear deformation and rotary inertia, and being $X$ and $Y$ the principal directions of orthotropy, is given by

$$
\begin{gathered}
D_{x} \frac{\partial^{4} w}{\partial^{4} x}+2 H \frac{\partial^{4} w}{\partial^{2} x \partial^{2} y}+D_{y} \frac{\partial^{4} w}{\partial^{4} y}+\rho h \frac{\partial^{2} w}{\partial^{2} t}=q_{z}(x, y, t) \\
q_{z}(x, y, t)=-\sum_{k=1}^{N_{P}}\left[\mathrm{H}\left(t-\frac{d_{k}}{V}\right)-\mathrm{H}\left(t-\frac{d_{k}+L}{V}\right)\right] P_{k} \delta\left(x-V t+d_{k}\right) \delta\left(y-y_{P}\right)
\end{gathered}
$$

where $D_{x}$ and $D_{y}$ are the flexural rigidities per unit of length $L$ or width $B$ of the plate with respect to the $X Z$ and $Y Z$ planes, respectively, $2 H=D_{1}+D_{2}+2\left(D_{x y}+D_{y x}\right), D_{1}$ and $D_{2}$ are the coupling flexural rigidities, $D_{x y}$ and $D_{y x}$ the torsional rigidities with respect to the same planes and $\rho$ the uniform mass density [20, 25]. In Eq. 1b, which represents the particular source of excitation of a train of $N_{P}$ constant 
moving loads $P_{k}$ travelling along $y=y_{P}$, parallel to the $X$ axis at constant speed $V$ (Fig. 2b), $\mathrm{H}$ and $\delta$ stand for the Heaviside unit step and the Dirac Delta functions, respectively. In what follows the free vibration

(a)

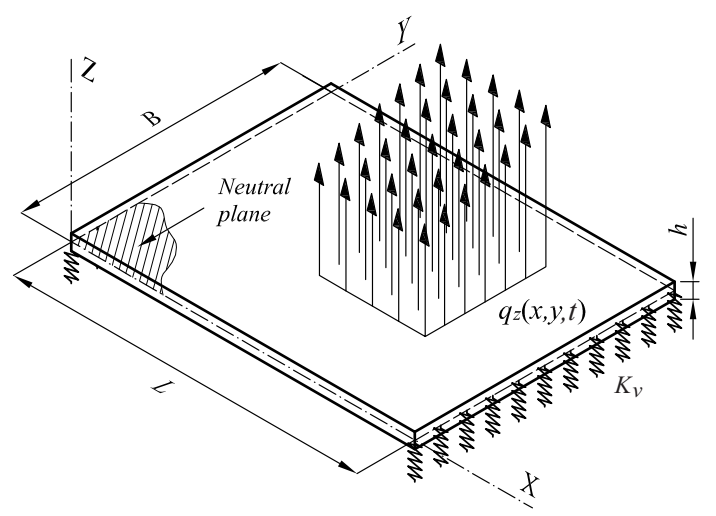

(b)

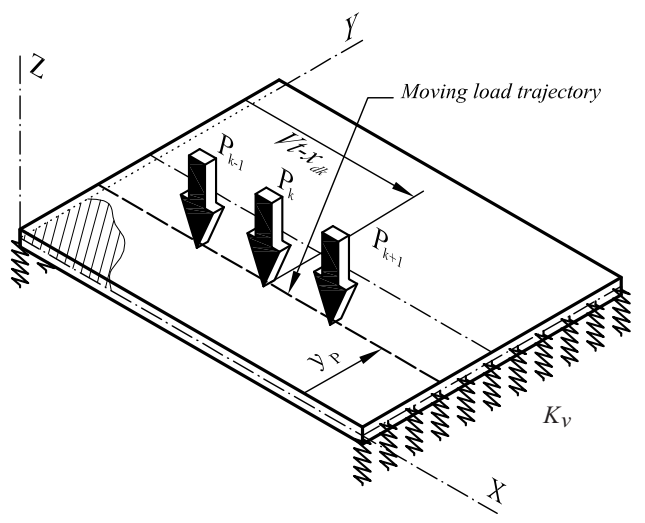

Figure 2: Rectangular Kirchhoff plate elastically-supported under (a) generic transverse load and (b) contant moving forces.

response induced by a single load traversing the plate parallel to the $X$ axis is formulated on (i) SS and (ii) ES boundary conditions.

\subsection{Plates simply-supported on two opposite borders}

Applying boundary conditions corresponding to two opposite sides SS and the other two borders free, the transverse displacement of the neutral plane $w(x, y, t)$ under the circulation of the moving loads may be expressed as a linear combination of the normal mode shapes $\Phi_{i j}(x, y)$

$$
w(x, y, t)=\sum_{i=1}^{N_{x}} \sum_{j=1}^{N_{y}} \Psi_{i j}(t) \Phi_{i j}(x, y)=\sum_{i=1}^{N_{x}} \sum_{j=1}^{N_{y}} \Psi_{i j}(t) \sin \left(\frac{i \pi x}{L}\right) Y_{i j}(y) C_{i j}
$$

where $\Psi_{i j}(t)$ stands for the $i j$-th modal amplitude, $C_{i j}$ depends on the mode normalization and $Y_{i j}(y)$ is provided in Appendix A (Eqs. A.1a A.1e). For the details of the derivation of the natural frequencies and mode shapes of a rectangular SS orthotropic plate the reader is referred to [25].

Substituting Eq. 2 in 1a, multiplying by the $m n$-th mode, integrating over the plate domain and in virtue of the modes orthogonality condition [25], the differential equation governing the $m n$-th modal amplitude in the absence of structural damping may be expressed as

$$
\ddot{\Psi}_{m n}(t)+\omega_{m n}^{2} \Psi_{m n}(t)=-\frac{1}{M_{m n}} \sum_{k=1}^{N_{p}}\left(H\left(t-\frac{d_{k}}{V}\right)-H\left(t-\frac{d_{k}+L}{V}\right)\right) P_{k} \Phi_{m n}\left(V t-d_{k}, y_{p}\right)
$$

where $M_{m n}$ stands for the $m n$-th modal mass and $\omega_{i j}$ is the circular frequency of the orthotropic plate (Eq A.2a in Appendix A). If only one load crosses the plate at a constant velocity, Eq. 3 presents the 
following solution

$$
\begin{array}{ll}
\Psi_{m n}(t)=-\frac{P C_{m n} Y_{m n}\left(y_{P}\right)}{M_{m n} \omega_{m n}^{2}} \frac{1}{1-K_{m n}^{2}}\left[\sin \left(K_{m n} \omega_{m n} t\right)-K_{m n} \sin \left(\omega_{m n} t\right)\right] & 0 \leq t \leq \frac{L}{V} \quad K_{m n} \neq 1 \\
\Psi_{m n}(t)=-\frac{P C_{m n} Y_{m n}\left(y_{P}\right)}{M_{m n} \omega_{m n}^{2}} \frac{1}{2}\left[\sin \left(\omega_{m n} t\right)-\omega_{m n} t \cos \left(\omega_{m n} t\right)\right] & 0 \leq t \leq \frac{L}{V} \quad K_{m n}=1
\end{array}
$$

where $K_{m n}$ is the non-dimensional speed parameter defined as

$$
K_{m n}=\frac{m \pi V}{\omega_{m n} L}
$$

Notice that in the previous equations $m$ corresponds to the number of half-sine waves travelled by the load when crossing the plate in the $m n$-th mode. As the load travels parallel to the $X$ axis, the modal deflection along the load path is always a sinusoidal function. When the load exits the plate, the latter is left in free vibration. The amplitude of this free vibration stage may be obtained solving Eq. 6 with initial conditions given by Eq. 4 at $t=L / V$.

$$
\ddot{\Psi}_{m n}(t)+\omega_{m n}^{2} \Psi_{m n}(t)=0 \quad t \geq L / V
$$

If the amplitude of the free vibrations is divided by the static solution when the load acts at $\left(L / 2, y_{P}\right), \Psi_{m n}^{s t}$, a non-dimensional amplitude in free vibration associated to a certain mode may be defined and expressed as

$$
R_{m n}=\frac{\Psi_{m n}}{\Psi_{m n}^{s t}}=\frac{K_{m n} \sqrt{2}}{1-K_{m n}^{2}} \sqrt{1-\cos (m \pi) \cos \left(\frac{m \pi}{K_{m n}}\right)} \quad \Psi_{m n}^{s t}=\left|-\frac{P C_{m n} Y_{m n}\left(y_{P}\right)}{M_{m n} \omega_{m n}^{2}}\right|
$$

In Fig. 3 the evolution of $R_{m n}$ with $K_{m n}$ is represented. $R_{m n}$ curves superimpose for modes with the same $m$ value. Maximum free vibration and cancellation conditions alternatively take place. Modes with the same $m$ value present identical non-dimensional speeds for maximum free vibration and cancellation (i.e. first longitudinal bending, first torsion and first transverse bending modes). Nevertheless, dimensional velocities will differ if natural frequencies are different. The particular conditions for maximum free vibration and cancellation are obtained and presented in section 2.3.

\subsection{Plates elastically-supported on two opposite borders}

Let us consider vertical elastic supports uniformly distributed along $x=0$ and $x=L$ (Fig. 2a). Let $K_{v}$ be the vertical stiffness constant per unit of length of the plate border. As in [10], a new parameter $\kappa$ is defined as the ratio between the longitudinal flexural stiffness of the plate and that of the vertical supports $\kappa=D_{x} B \pi^{3} / \bar{K}_{v} L^{3}$, where $\bar{K}_{v}$ is the resultant vertical stiffness along one border of the plate. Following Leissa's approach [26], the plate transverse displacement may be approximated as a linear combination of the product of two functions $X_{i}(x)$ and $Y_{j}(y)$.

$$
w(x, y, t)=\sum_{i=1}^{N_{x}} \sum_{j=1}^{N_{y}} \Psi_{i j}(t) \Phi_{i j}(x, y)=\sum_{i=1}^{N_{x}} \sum_{j=1}^{N_{y}} \Psi_{i j}(t) X_{i}(x) Y_{j}(y) C_{i j}
$$



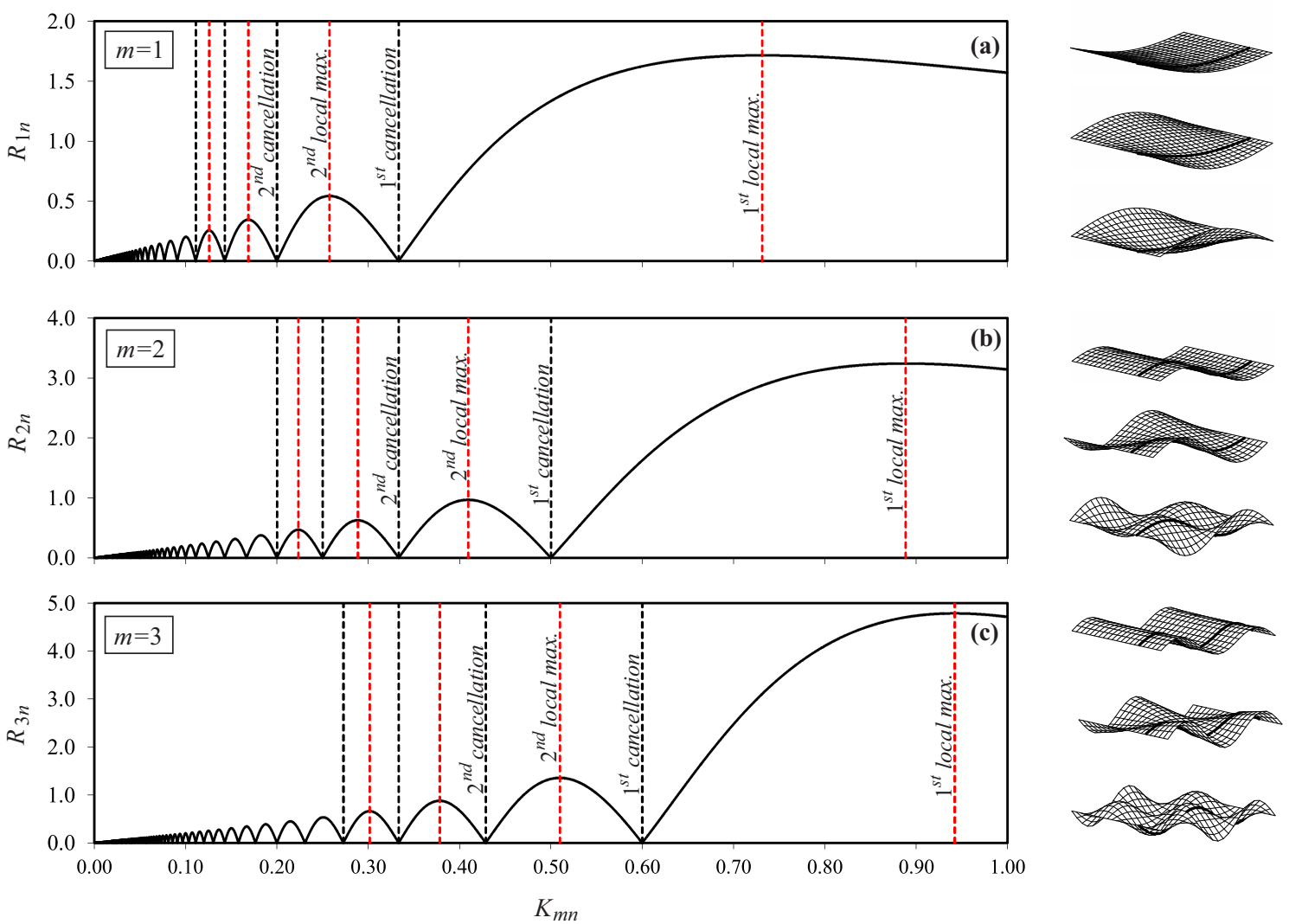

Figure 3: $R_{m n}$ versus non-dimensional speed $K_{m n}$ in SS plates for modes with one, two and three half-sine waves along the load path $(m=1,2,3)$.

where $X_{i}(x)$ and $Y_{j}(y)$ are approximated as the mode shapes of the associated undamped beams having the same boundary conditions as the plate in the $X$ and $Y$ directions, respectively. For the details of the formulation the reader is referred to Appendix A (Eqs. A.3 to A.6.

Following the same approach as in the SS case, in virtue of the modes orthogonality condition and neglecting structural damping, one concludes that Eq. 3 governs again the ES plate $m n$-th modal amplitude under the circulation of a single load travelling at constant speed, with the only difference that now $\Phi_{m n}$ stands for the ES plate mode. As the load moves parallel to the longitudinal axis, admitting the approximation of the ES plate modes as the product of the corresponding beams modes, the modal load time variation is proportional to that of a ES beam subjected to the same loading conditions. The solution for the forced vibration of a Bernoulli-Euler ES beam under a single load was first presented in a compact format by Museros et al. and may be consulted in [10]. From the modal amplitude and its derivative at the time instant when the load exits the plate, the $m n$-th modal response in free vibration may be obtained. Finally, the modal amplitude in free vibration can be defined as in the SS plate case applying a proper normalization 


$$
R_{m n}=\frac{\Psi_{m n}}{\Psi_{m n}^{s t}}=f\left(\bar{K}_{m n}\right) \quad \Psi_{m n}^{s t}=\left|-\frac{P C_{m n} Y_{m n}\left(y_{P}\right)}{M_{m n} \bar{\omega}_{m n}^{2}}\right|
$$

For clarification, an overbar is used in what follows to differentiate magnitudes corresponding to the ES plate. In Eq. $9 \bar{K}_{m n}$ is defined as the ratio between the forcing frequency of the load and the ES plate circular frequency, $\bar{\omega}_{m n}^{2}$ (EqA.6 in Appendix A. In order to be able to compare $R_{m n}$ curves for different support conditions, the following relation between $\bar{K}_{m n}$ and $K_{m n}$ is admitted between the ES and the SS plate, even though it strictly applies to the beam case:

$$
\bar{K}_{m n}=\frac{\bar{\lambda}_{m} V}{\bar{\omega}_{m n} L} \simeq \frac{m \pi}{\bar{\lambda}_{m}} K_{m n}
$$

When $R_{m n}$ is plotted vs $\bar{K}_{m n}$ in the ES plate case a similar evolution to that shown in Fig. 3 is observed, where cancellation and maximum free vibration conditions alternate as the velocity increases. In the next subsection these values are computed for SS and ES boundary conditions.

\subsection{Non-dimensional speeds for maximum modal response and cancellation}

In the previous section it was shown that the amplification of the free vibrations in SS or ES orthotropic rectangular plates after the circulation of a single load can be expressed in terms of only one parameter, the non-dimensional speed $K_{m n}$. From Eqs. 7 and 9 it is possible to obtain the conditions for maximum free vibration and cancellation in each mode as

$$
R_{m n}\left(K_{m n}\right)=0 \Longrightarrow K_{m n}^{c i} \quad \frac{\partial R_{m n}\left(K_{m n}\right)}{\partial K_{m n}}=0 \Longrightarrow K_{m n}^{\max , i}
$$

In Eq. $11 K_{m n}^{c i}$ for $i=1,2,3, \ldots$ stands for the values of $K_{m n}$ of cancellation as the velocity decreases (i.e. $K_{m n}^{c 1}$ is the first cancellation taking place at the highest speed). Likewise, $K_{m n}^{\max , i}$ designates the nondimensional speeds leading to a local maximum of the plate free vibrations, corresponding $K_{m n}^{\max , 1}$ to the local maximum taking place at the highest speed. In Fig. 3, first and second cancellation speeds and local maxima are indicated for the SS plate modes with $m=1,2,3$. In Table 1 the values for the first three cancellation and maximum free vibration speeds are included for modes with $m=1, \kappa=0$ (SS case) and $\kappa=[0.05,0.1]$ (ES case). Only the values associated to $m=1$ are included as the main focus of this study is the response of girder or slab decks associated to the first longitudinal bending, first torsion and first transverse bending modes, which presumably will have the highest contribution to the overall transverse response for frequencies up to $30 \mathrm{~Hz}$. Notice that these values coincide with those of the SS and the ES beams [10] due to the expression for the load in modal coordinates. In section 4, these ratios are compared to real cancellation and maximum free vibration conditions computed numerically, and their accuracy is evaluated within a catalogue of realistic structures. 


\begin{tabular}{|c|c|c|c|c|c|c|c|c|}
\hline \multirow{2}{*}{$\kappa$} & \multicolumn{4}{|l|}{$K_{1 n}^{c i}$} & \multicolumn{4}{|l|}{$K_{1 m}^{\max , i}$} \\
\hline & $i=1$ & $i=2$ & $i=3$ & $i=4$ & $i=1$ & $i=2$ & $i=3$ & $i=4$ \\
\hline 0.0 & 0.3333 & 0.2000 & 0.1429 & 0.1111 & 0.7314 & 0.2576 & 0.1687 & 0.1258 \\
\hline 0.05 & 0.3323 & 0.1997 & 0.1427 & 0.1109 & 0.7288 & 0.2563 & 0.1684 & 0.1254 \\
\hline 0.10 & 0.3312 & 0.1991 & 0.1419 & 0.1100 & 0.7242 & 0.2556 & 0.1673 & 0.1245 \\
\hline
\end{tabular}

Table 1: Analytical values for the first four cancellation and maximum free vibration speeds.

\section{Forced vibrations of orthotropic rectangular plates under a series of moving loads: maxi-} mum resonance and cancellation of resonance

When a beam or a plate is traversed by a series of equidistant loads moving at constant speed, the excitation may induce resonance on it. Resonance can occur when the time interval between the passage of loads sequences separated a characteristic distance $d_{k}$ is a multiple of a natural period de the structure. The conditions for resonance in a SS (Eq. 12a) or an ES (Eq. 12b) rectangular plate can be formulated in a dimensional or non-dimensional format as

$$
\begin{aligned}
& V_{m n}^{r}=\frac{d_{k} f_{m n}}{j} \rightarrow K_{m n}^{r}=\frac{m}{2 j} \frac{d_{k}}{L} \\
& V_{m n}^{r}=\frac{d_{k} \bar{f}_{m n}}{j} \rightarrow K_{m n}^{r} \simeq \frac{\bar{\lambda}_{m}}{m \pi} \bar{K}_{m n}^{r}=\left(\frac{\bar{\lambda}_{m}}{\pi}\right)^{2} \frac{1}{2 m j} \frac{d_{k}}{L}
\end{aligned}
$$

where $j$ is the resonance order, or number of oscillations that the plate undergoes in a particular mode between the passage of two consecutive loads separated a distance $d_{k}$. At resonance, the free vibrations that every single axle leaves on the structure add in phase and the plate response progressively increases. Consequently, the amplitude of the free vibrations at the particular resonant speed, will determine to an important extent the prominence of the resonant peak. When a resonant speed coincides or is close to a maximum free vibration condition, an important amplification of the response should be expected. On the contrary, as it approaches to a cancellation condition, it will become almost imperceptible. Based on the previous, the conditions for resonance and either maximum free vibration or cancellation are equated, and $L / d_{k}$ ratios for maximum resonance or cancellation of it are extracted both for the SS (Eq. 13) and the ES (Eq. 14) plates:

$$
\begin{gathered}
K_{m n}^{r}=K_{m n}^{c i} \rightarrow\left(\frac{L}{d_{k}}\right)_{m j}^{c i}=\frac{m}{2 j} \frac{1}{K_{m n}^{c i}} \\
K_{m n}^{r}=K_{m n}^{\max , i} \rightarrow\left(\frac{L}{d_{k}}\right)_{m j}^{m a x, i}=\frac{m}{2 j} \frac{1}{K_{m n}^{\max , i}}
\end{gathered}
$$




$$
\begin{gathered}
K_{m n}^{r}=K_{m n}^{c i} \rightarrow\left(\frac{L}{d_{k}}\right)_{m j}^{c i}=\left(\frac{\bar{\lambda}_{m}}{\pi}\right)^{2} \frac{1}{2 m j} \frac{1}{K_{m n}^{c i}} \\
K_{m n}^{r}=K_{m n}^{\max , i} \rightarrow\left(\frac{L}{d_{k}}\right)_{m j}^{\max , i}=\left(\frac{\bar{\lambda}_{m}}{\pi}\right)^{2} \frac{1}{2 m j} \frac{1}{K_{m n}^{\max , i}}
\end{gathered}
$$

In Table $2, L / d_{k}$ ratios for maximum resonance and cancellation of resonance are included for $m=1$, $\kappa=[0.00,0.05,0.10]$, first four resonant orders $j$, and first four cancellation or local maxima events $i$. The ratios are computed by substitution of the results in Table 1 into Eqs. 13 and 14 . These values coincide with those of SS and ES beams presented in [10, as it is justified through the derivations presented in section 2 In section 4 , the accuracy of analytical $K_{1 n}^{c i}$ and $K_{1 n}^{\max , i}$ from Table 1 and, therefore that of the $L / d_{k}$ ratios

\begin{tabular}{|c|c|c|c|c|c|c|c|c|}
\hline \multirow{2}{*}{$j$} & \multirow{2}{*}{$\begin{array}{l}\left(L / d_{k}\right)_{1 j}^{c i} \\
i=1\end{array}$} & \multicolumn{3}{|l|}{$\kappa=0$} & \multirow{2}{*}{$\begin{array}{l}\left(L / d_{k}\right)_{1 j}^{\max , i} \\
i=1\end{array}$} & \multicolumn{3}{|l|}{$\kappa=0$} \\
\hline & & $i=2$ & $i=3$ & $i=4$ & & $i=2$ & $i=3$ & $i=4$ \\
\hline 1 & 1.5000 & 2.5000 & 3.5000 & 4.5000 & 0.6836 & 1.9411 & 2.9640 & 3.9737 \\
\hline 2 & 0.7500 & 1.2500 & 1.7500 & 2.2500 & 0.3418 & 0.9705 & 1.4820 & 1.9869 \\
\hline 3 & 0.5000 & 0.8333 & 1.1667 & 1.5000 & 0.2279 & 0.6470 & 0.9880 & 1.3246 \\
\hline 4 & 0.3750 & 0.6250 & 0.8750 & 1.1250 & 0.1709 & 0.4853 & 0.7410 & 0.9934 \\
\hline \multirow{2}{*}{$j$} & $\left(L / d_{k}\right)_{1 j}^{c i}$ & \multicolumn{3}{|l|}{$\kappa=0.05$} & $\left(L / d_{k}\right)_{1 j}^{\max , i}$ & \multicolumn{3}{|l|}{$\kappa=0.05$} \\
\hline & $i=1$ & $i=2$ & $i=3$ & $i=4$ & $i=1$ & $i=2$ & $i=3$ & $i=4$ \\
\hline 1 & 1.4582 & 2.4263 & 3.3966 & 4.3689 & 0.6650 & 1.8864 & 2.8787 & 3.8605 \\
\hline 2 & 0.7291 & 1.2132 & 1.6983 & 2.1844 & 0.3325 & 0.9432 & 1.4394 & 1.9302 \\
\hline 3 & 0.4861 & 0.8088 & 1.1322 & 1.4563 & 0.2217 & 0.6288 & 0.9596 & 1.2868 \\
\hline 4 & 0.3646 & 0.6066 & 0.8491 & 1.0922 & 0.1662 & 0.4716 & 0.7197 & 0.9651 \\
\hline \multirow{2}{*}{$j$} & $\left(L / d_{k}\right)_{1 j}^{c i}$ & \multicolumn{3}{|l|}{$\kappa=0.10$} & $\left(L / d_{k}\right)_{1 j}^{\max , i}$ & \multicolumn{3}{|l|}{$\kappa=0.10$} \\
\hline & $i=1$ & $i=2$ & $i=3$ & $i=4$ & $i=1$ & $i=2$ & $i=3$ & $i=4$ \\
\hline 1 & 1.4195 & 2.3620 & 3.3133 & 4.2731 & 0.6493 & 1.8394 & 2.8101 & 3.7761 \\
\hline 2 & 0.7098 & 1.1810 & 1.6567 & 2.1366 & 0.3246 & 0.9197 & 1.4051 & 1.8880 \\
\hline 3 & 0.4732 & 0.7873 & 1.0440 & 1.4244 & 0.2164 & 0.6131 & 0.9367 & 1.2587 \\
\hline 4 & 0.3549 & 0.5905 & 0.8283 & 1.0683 & 0.1623 & 0.4599 & 0.7025 & 0.9440 \\
\hline
\end{tabular}
included in Table 2, is evaluated in the case of 112 real bridges with skewed geometries.

Table 2: $L / d_{k}$ ratios leading to cancellation of resonance and maximum resonance for modes with $m=1$ and $\kappa=[0,0.05,0.10]$. 


\section{Sensitivity analysis on plate obliquity and supports vertical flexibility}

\subsection{Methodology}

In order to evaluate the applicability of the previously derived analytical ratios, first, an extensive bridge catalogue is designed covering lengths from 10 to $25 \mathrm{~m}$, considering longitudinal flexural stiffnesses representative of both existing structures belonging to conventional lines and new stiffer bridges specifically designed for HS lines, accounting for obliquity levels in the range $0^{\circ}$ to $45^{\circ}$ and envisaging the absence or presence of neoprene bearings at the supports. A total of 112 bridges are pre-dimensioned and evaluated. In sections 4.1.1, 4.1.2 and 4.1.3 the specific properties of the bridges under study, the main assumptions of the numerical model and the types of analyses performed are described.

\subsubsection{Definition of a bridge catalogue}

A representative ensemble of double-track, simply-supported pre-stressed concrete girder decks has been designed with the purpose of covering the mechanical properties of most existing bridges belonging to conventional and high-speed railway lines of this typology. Among the different bridge typologies that can be found in short-to-medium span lengths, precast decks composed by double-T girders have been selected due to their low intrinsic torsional stiffness which makes their dynamic behaviour significantly differ from that of a beam-type structure, exhibiting a noticeable contribution of the first torsion and first transverse bending modes to the vertical acceleration response under railway excitation [24]. Even though in new railway lines in the range of lengths of interest these bridges have been replaced by stiffer solutions with better dynamic performance (i.e. twin cell box girder decks), in the first HS Spanish line, Madrid-Sevilla, several double-T girder bridges exist (see Fig. 4), and many may be found in conventional lines as well. This typology usually covers span lengths between $10 \mathrm{~m}$ and $25 \mathrm{~m}$ with slenderness ratios (depth/span) no higher than $1 / 13$.

Fig. 5 and Table 3 show, respectively, the cross section and main properties of the bridge catalogue. Considered span lengths range from 10 to $25 \mathrm{~m}$ in $2.5 \mathrm{~m}$ intervals. Two longitudinal flexural stiffness ratios $L / \delta$ (span length/maximum vertical displacement under static UIC-71 load model from Eurocode 1 [27]) are evaluated for each span: $L / \delta$ ratios in the range [2000-3000], which are more frequent in conventional lines (from now on $L / \delta_{2000}$ bridges), and $L / \delta>3000$ for HS lines (from now on $L / \delta_{3000}$ bridges). The number of longitudinal girders of the decks, $N_{g}$, is also provided in Table 3 . Bridges with 5 or 6 girders have been pre-dimensioned in each case. The nominal value for the concrete strength $f_{c k}$ assumed for the upper slab is $30 \mathrm{MPa}$; for the longitudinal girders a value of $f_{c k}=50 \mathrm{MPa}$ is considered. In a first approach, the stiffening effect of hypothetical cross beams at the end sections is not included, as (i) these elements are not always present or may not be properly incorporated; and (ii) in previous works by the authors [28] it has been shown that the effect of these elements on the first three modal shapes of this type of bridges 

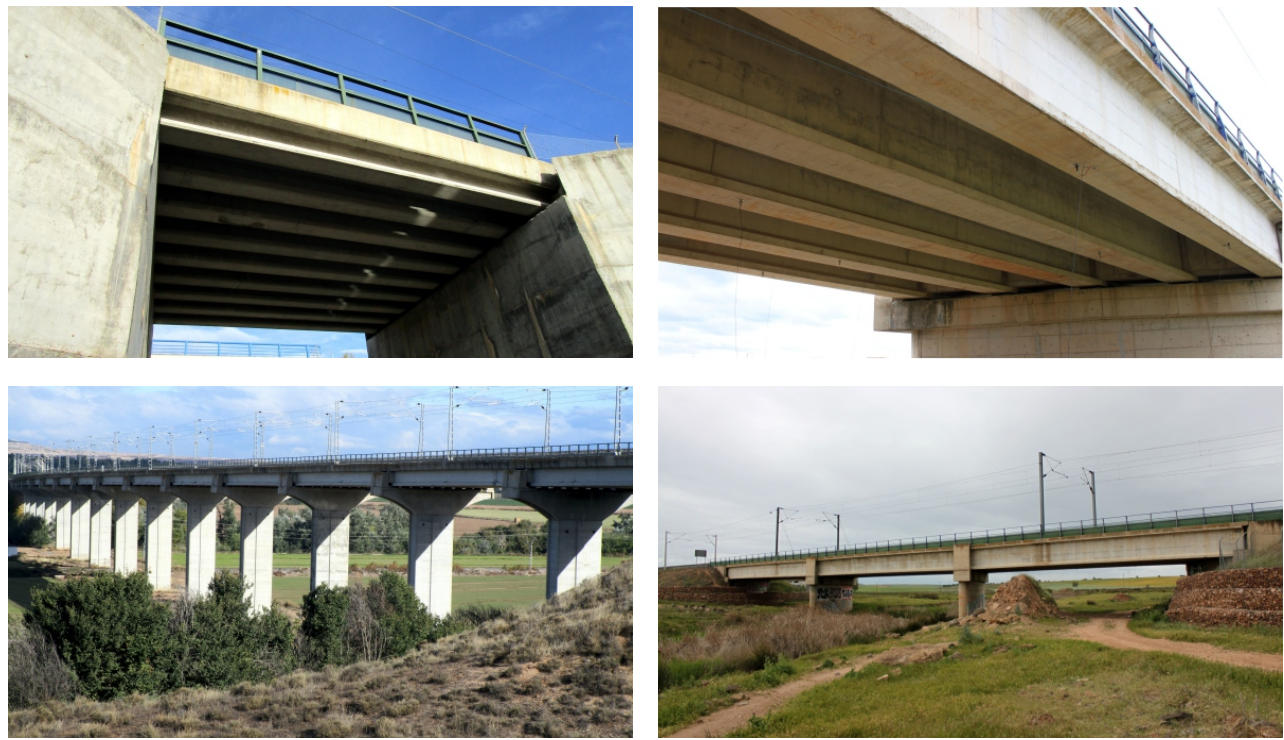

Figure 4: Precast pre-stressed double-T girder decks in Madrid-Sevilla HS line.

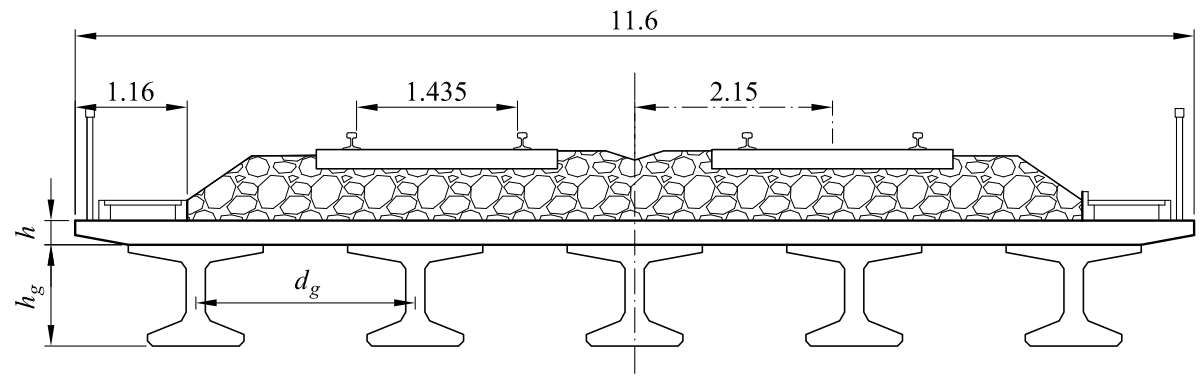

Figure 5: Intermediate cross section of the girder bridges of study.

is limited. Regarding the vertical support of the girders, two possibilities are envisaged: rigid supports (SS deck) and flexible supports, accounting for the neoprene bearings vertical stiffness (ES deck). In the ES case the relative flexibility of the bearings with respect to the flexural flexibility of the plate assumed is a reasonable and common estimate leading to a decrease of the fundamental frequency of the bridges of approximately $3-4 \%(\kappa=0.05)$. Furthermore, four obliquity levels for each deck are analysed: $0^{\circ}, 15^{\circ}$, $30^{\circ}$ and $45^{\circ}$.

In Fig. 6 the first three natural frequencies of the bridges under study are represented. The natural frequencies are computed from a modal analysis using the numerical model described in section 4.1.2 The first three mode shapes in frequency order of all the bridges that conform the catalogue are the first longitudinal bending, first torsion and first transverse bending modes. The evolution of these three natural frequencies with the level of obliquity is presented in section 4.2.1. Solid markers correspond to bridges with 


\begin{tabular}{|c|c|c|c|c|c|c|c|c|c|}
\hline \multirow{2}{*}{ Span $L[\mathrm{~m}]$} & \multirow{2}{*}{$h[\mathrm{~m}]$} & \multicolumn{4}{|c|}{ Longitudinal girders } & \multirow{2}{*}{$\begin{array}{c}\text { Mass } \\
{[\mathrm{kg} / \mathrm{m}]}\end{array}$} & \multirow{2}{*}{$\begin{array}{c}\text { Supports } \\
\kappa\end{array}$} & \multirow{2}{*}{$L / \delta$} & \multirow{2}{*}{ BrName } \\
\hline & & $N_{g}$ & $d_{g}[\mathrm{~m}]$ & $h_{g}[\mathrm{~m}]$ & $I_{h}\left[\mathrm{~m}^{4}\right]$ & & & & \\
\hline \multirow[t]{2}{*}{10} & 0.22 & 6 & 2.0 & 0.6 & 0.01098 & 18765 & $0(\mathrm{SS}), 0.05$ & 2119 & B100.2000.6 \\
\hline & 0.22 & 5 & 2.275 & 0.65 & 0.02150 & 20525 & $0(\mathrm{SS}), 0.05$ & 3320 & B100.3000.5 \\
\hline \multirow[t]{2}{*}{12.5} & 0.25 & 6 & 2.0 & 0.8 & 0.02278 & 19995 & 0(SS), 0.05 & 2229 & B125.2000.6 \\
\hline & 0.22 & 5 & 2.0 & 0.85 & 0.04339 & 20975 & $0(\mathrm{SS}), 0.05$ & 3148 & B125.3000.5 \\
\hline \multirow[t]{2}{*}{15} & 0.25 & 6 & 2.0 & 1.0 & 0.03961 & 20355 & $0(\mathrm{SS}), 0.05$ & 2154 & B150.2000.6 \\
\hline & 0.25 & 5 & 2.275 & 1.05 & 0.07423 & 22295 & $0(\mathrm{SS}), 0.05$ & 3226 & B150.3000.5 \\
\hline \multirow[t]{2}{*}{17.5} & 0.25 & 6 & 2.0 & 1.2 & 0.06195 & 20715 & $0(\mathrm{SS}), 0.05$ & 2090 & B175.2000.6 \\
\hline & 0.25 & 5 & 2.275 & 1.25 & 0.11476 & 22745 & $0(\mathrm{SS}), 0.05$ & 3079 & B175.3000.5 \\
\hline \multirow[t]{2}{*}{20} & 0.25 & 6 & 2.0 & 1.4 & 0.09029 & 21075 & $0(\mathrm{SS}), 0.05$ & 2031 & B200.2000.6 \\
\hline & 0.25 & 6 & 2.0 & 1.45 & 0.16571 & 24590 & $0(\mathrm{SS}), 0.05$ & 3351 & B200.3000.6 \\
\hline \multirow[t]{2}{*}{22.5} & 0.25 & 6 & 2.0 & 1.6 & 0.1251 & 21435 & $0(\mathrm{SS}), 0.05$ & 1977 & B225.2000.6 \\
\hline & 0.25 & 5 & 2.275 & 1.65 & 0.23652 & 24396 & $0(\mathrm{SS}), 0.05$ & 3250 & B225.3000.5 \\
\hline \multirow[t]{2}{*}{25} & 0.25 & 6 & 2.0 & 1.7 & 0.21812 & 24302 & $0(\mathrm{SS}), 0.05$ & 2320 & B250.2000.6 \\
\hline & 0.25 & 5 & 2.275 & 1.85 & 0.131500 & 24896 & $0(\mathrm{SS}), 0.05$ & 3126 & B250.3000.5 \\
\hline
\end{tabular}

Table 3: Bridge catalogue definition for a particular obliquity level.

infinitely rigid supports and voided markers to the ES decks. Also, squares represent natural frequencies of the most flexible predesigned bridges for each length, while circles are used for bridges with higher flexural stiffness, representative of new structures which may exist in HS railway lines. Finally, in Fig. 6(a) the upper and lower limits of the fundamental frequency for simplified analysis according to Eurocode 1 [27] are indicated.

\subsubsection{Numerical model description}

The dynamic response of the previously described bridges under railway traffic is obtained numerically in the time domain. The main features of the numerical model, sketched in Fig.7, are the following:

- An orthotropic thin plate is used to calculate the deck dynamic response under railway traffic. The plate is discretised in $C^{1}$ compatible linear varying curvature triangular finite elements with 12 degrees of freedom [29].

- The weight of the dead loads (ballast, sleepers, rails, sidewalks and handrails) is uniformly distributed over the plate. No additional rigidity from the rail components is considered in a first approach.

- The laminated rubber bearings of the deck girders are included in the model as an equivalent vertical stiffness uniformly distributed along the supports lengths. In a previous publication the authors have 

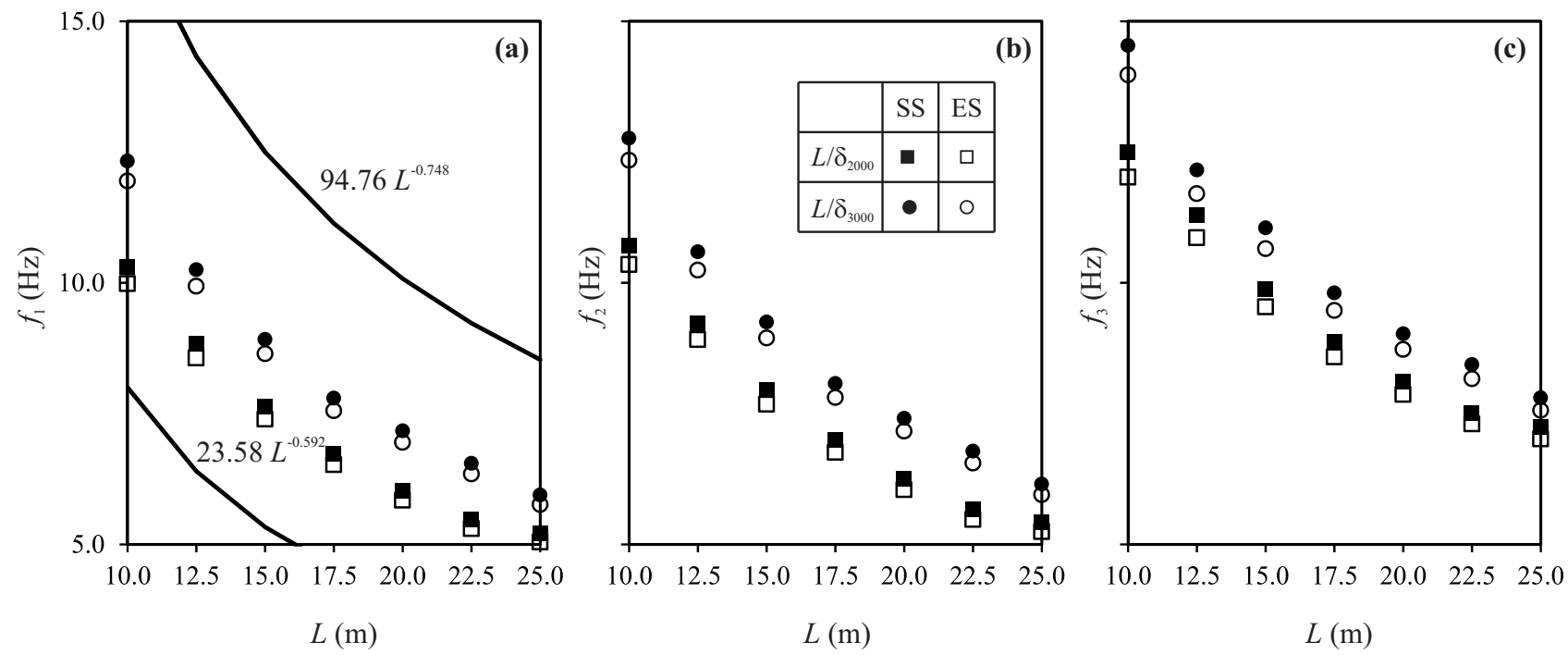

Figure 6: First three natural frequencies of the bridges under study in the straight configurations $(\alpha=0)$.

proven that the uniform distribution of the girders and bearings vertical stiffness admitted in the orthotropic plate model is not determinant for the calculation of the maximum response at the deck intermediate sections for moderate frequency contents [7].

- Constant moving loads represent the railway excitation, therefore neglecting vehicle-structure interaction effects. As the track rigidity is not included in the numerical model, when a load enters or exits the bridge crossing a border element, a transient phenomenon takes place due to the presence of the elastic bearings that leads to unrealistic high-frequency modal contributions of the plate. To overcome this numerical problem, the load distributive effect of rails, sleepers and ballast is simulated in the model at the abutments vicinity. To this end, the value of each axle load is modulated throughout a load-print distributive function centred on the wheel position and based on the Zimmerman-Timoshenko solution for an infinite beam on a Winkler foundation [30].

- The dynamic equations of motion are transformed into modal space and numerically integrated in the time domain applying the Newmark- $\beta$ linear acceleration algorithm.

The orthotropic plate constants, $D_{x}, D_{y}, D_{1}, D_{2}, D_{x y}$ and $D_{y x}$, are computed for each of the bridges of the catalogue from its geometry and material data. The calculated values for these constants are given in Table 4. For further details on this step the reader is referred to the classical reference [31]. 


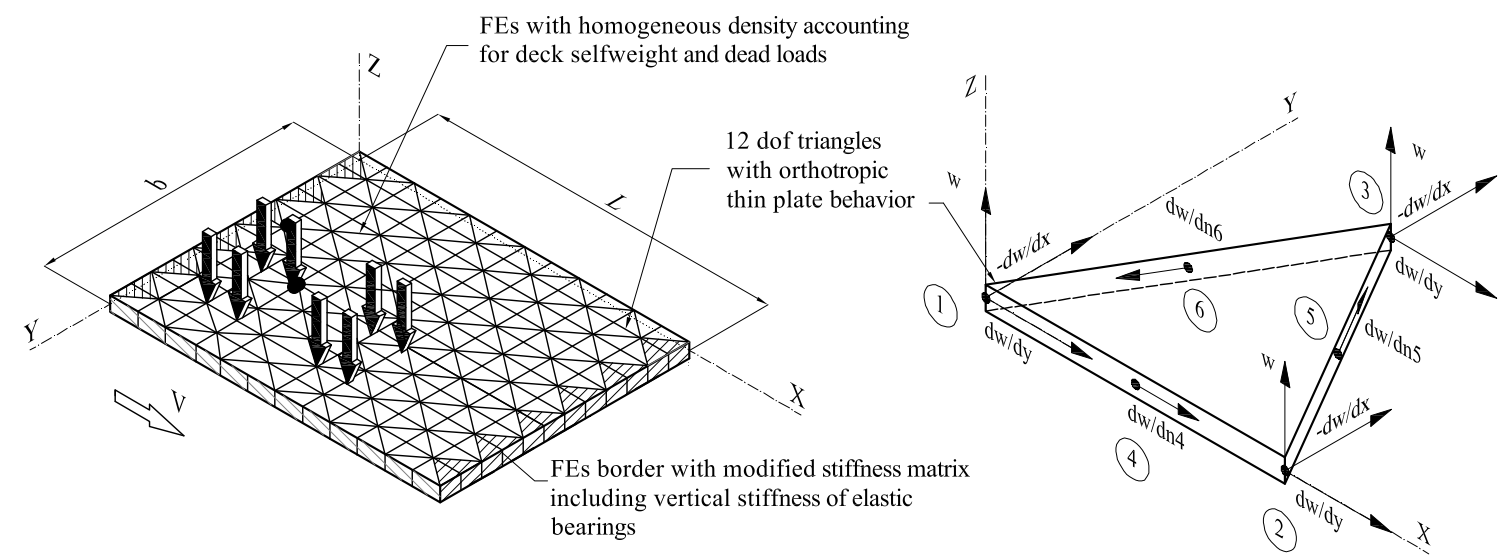

Figure 7: Orthotropic plate FE model.

\subsubsection{Numerical analyses performed}

First, the modal properties of the bridges under study are obtained with the aim of evaluating the evolution of the lowest natural frequencies with the deck obliquity and the supports vertical flexibility. Second, the free vibration response under the circulation of a single load of all the bridges is computed, and speeds for cancellation and maximum free vibration are extracted for the first three modes. Results are compared with the analytical predictions given in Table 1, and errors when using these predictions in skewed and ES decks are delimited. Finally, the effect of structural damping is shown on a subset of short bridges.

\subsection{Sensitivity analysis results}

\subsubsection{Evolution of modal parameters}

In Fig. 8 the variations of the first three natural frequencies of the bridges under study with respect to their homologous in the straight case $(\alpha=0)$ are represented. Figures $8(\mathrm{a})$-(b), $8(\mathrm{c})$-(d) and $8(\mathrm{e})$-(f) represent the variations of the first bending, first torsion and first transverse bending frequencies, respectively. The left column plots correspond to the SS case $(\kappa=0.0)$ and the right column ones to the ES case for $\kappa=0.05$. Finally, the results of the less stiff bridges $\left(L / \delta_{2000}\right)$ are indicated with solid traces and those of bridges with highest flexural stiffness $\left(L / \delta_{3000}\right)$, are indicated with solid circles.

From the analyses of these results it can be concluded that (i) the level of obliquity leads to an increase in the decks natural frequencies with a highest impact on the shortest spans $(10 \mathrm{~m})$, the bridges with lowest flexural stiffness $\left(L / \delta_{2000}\right)$ and the highest skew angle $\left(45^{\circ}\right)$; (ii) the previous tendency occurs both in SS and ES structures, although in the latter case the increments are slightly inferior; (iii) frequency variations increase with the mode number. The maximum detected variations reach $6 \%, 10 \%$ and $18 \%$ for the fundamental, first torsion and first transverse bending modes, respectively; (iv) as the length increases, 


\begin{tabular}{cccccc}
\hline BrName & $D_{x}[\mathrm{Nm}]$ & $D_{y}[\mathrm{Nm}]$ & $D_{1,2}[\mathrm{Nm}]$ & $D_{x y}[\mathrm{Nm}]$ & $D_{y x}[\mathrm{Nm}]$ \\
\hline B100.2000.6 & $6.960 \mathrm{E} 8$ & $3.092 \mathrm{E} 7$ & $6.183 \mathrm{E} 6$ & $3.877 \mathrm{E} 7$ & $2.473 \mathrm{E} 7$ \\
B100.3000.5 & $1.090 \mathrm{E} 9$ & $3.092 \mathrm{E} 7$ & $6.183 \mathrm{E} 6$ & $6.721 \mathrm{E} 7$ & $2.473 \mathrm{E} 7$ \\
B125.2000.6 & $1.332 \mathrm{E} 9$ & $4.537 \mathrm{E} 7$ & $9.073 \mathrm{E} 6$ & $5.128 \mathrm{E} 7$ & $3.629 \mathrm{E} 7$ \\
B125.3000.5 & $1.880 \mathrm{E} 9$ & $3.092 \mathrm{E} 7$ & $6.183 \mathrm{E} 6$ & $6.977 \mathrm{E} 7$ & $2.473 \mathrm{E} 7$ \\
B150.2000.6 & $2.096 \mathrm{E} 9$ & $4.537 \mathrm{E} 7$ & $9.073 \mathrm{E} 6$ & $5.224 \mathrm{E} 7$ & $3.629 \mathrm{E} 7$ \\
B150.3000.5 & $3.138 \mathrm{E} 9$ & $4.537 \mathrm{E} 7$ & $9.073 \mathrm{E} 6$ & $8.401 \mathrm{E} 7$ & $3.629 \mathrm{E} 7$ \\
B175.2000.6 & $3.075 \mathrm{E} 9$ & $4.537 \mathrm{E} 7$ & $9.073 \mathrm{E} 6$ & $5.319 \mathrm{E} 7$ & $3.629 \mathrm{E} 7$ \\
B175.3000.5 & $4.530 \mathrm{E} 9$ & $4.537 \mathrm{E} 7$ & $9.073 \mathrm{E} 6$ & $8.670 \mathrm{E} 7$ & $3.629 \mathrm{E} 7$ \\
B200.2000.6 & $4.285 \mathrm{E} 9$ & $4.537 \mathrm{E} 7$ & $9.073 \mathrm{E} 6$ & $5.415 \mathrm{E} 7$ & $3.629 \mathrm{E} 7$ \\
B200.3000.6 & $7.069 \mathrm{E} 9$ & $4.537 \mathrm{E} 7$ & $9.073 \mathrm{E} 6$ & $1.000 \mathrm{E} 8$ & $3.629 \mathrm{E} 7$ \\
B225.2000.6 & $5.742 \mathrm{E} 9$ & $4.537 \mathrm{E} 7$ & $9.073 \mathrm{E} 6$ & $5.510 \mathrm{E} 7$ & $3.629 \mathrm{E} 7$ \\
B225.3000.5 & $9.368 \mathrm{E} 9$ & $4.537 \mathrm{E} 7$ & $9.073 \mathrm{E} 6$ & $1.143 \mathrm{E} 8$ & $3.629 \mathrm{E} 7$ \\
B250.2000.6 & $8.984 \mathrm{E} 9$ & $4.537 \mathrm{E} 7$ & $9.073 \mathrm{E} 6$ & $1.050 \mathrm{E} 8$ & $3.629 \mathrm{E} 7$ \\
B250.3000.5 & $1.201 \mathrm{E} 10$ & $4.537 \mathrm{E} 7$ & $9.073 \mathrm{E} 6$ & $1.180 \mathrm{E} 8$ & $3.629 \mathrm{E} 7$ \\
\hline
\end{tabular}

Table 4: Plate models orthotropic constants corresponding to bridges of the catalogue.

the effect of the deck obliquity diminishes, falling under $10 \%$ for the longest spans in all the cases.

\subsubsection{Free vibration under a single load moving at constant speed}

The free vibration response of the bridges under study after the passage of a constant moving load is analysed in this section. The objectives are to (i) investigate if cancellation and maximum free vibration speeds occur and alternate as in the analytical straight SS case (subsection 2.3); and (ii) verify the adequacy of the ratios provided in Table 1 to predict these phenomena with the increase of the plate obliqueness. Finally, conclusions regarding the effect of structural damping are provided.

First, the free vibration response of the 112 bridges under study after the circulation of a single load is obtained in the absence of damping. The load travels in all the cases with the same eccentricity $(1 / 4$ of the deck width). In the SS case $(\kappa=0)$ and for the straight configurations $(\alpha=0)$ this response coincides with that shown in Fig. 3(a). The maximum modal displacement in the first three modes is obtained for several travelling speeds. The range of speeds considered is such that the first cancellation and second maximum of the free vibrations are reached and a sufficiently small velocity increment is selected to obtain these two conditions with accuracy $\left(\Delta V=10^{-4} \mathrm{~m} / \mathrm{s}\right)$. The second maximum is evaluated as the first one will correspond to an unrealistic high speed with existing bridges and railway convoys. Finally the errors 

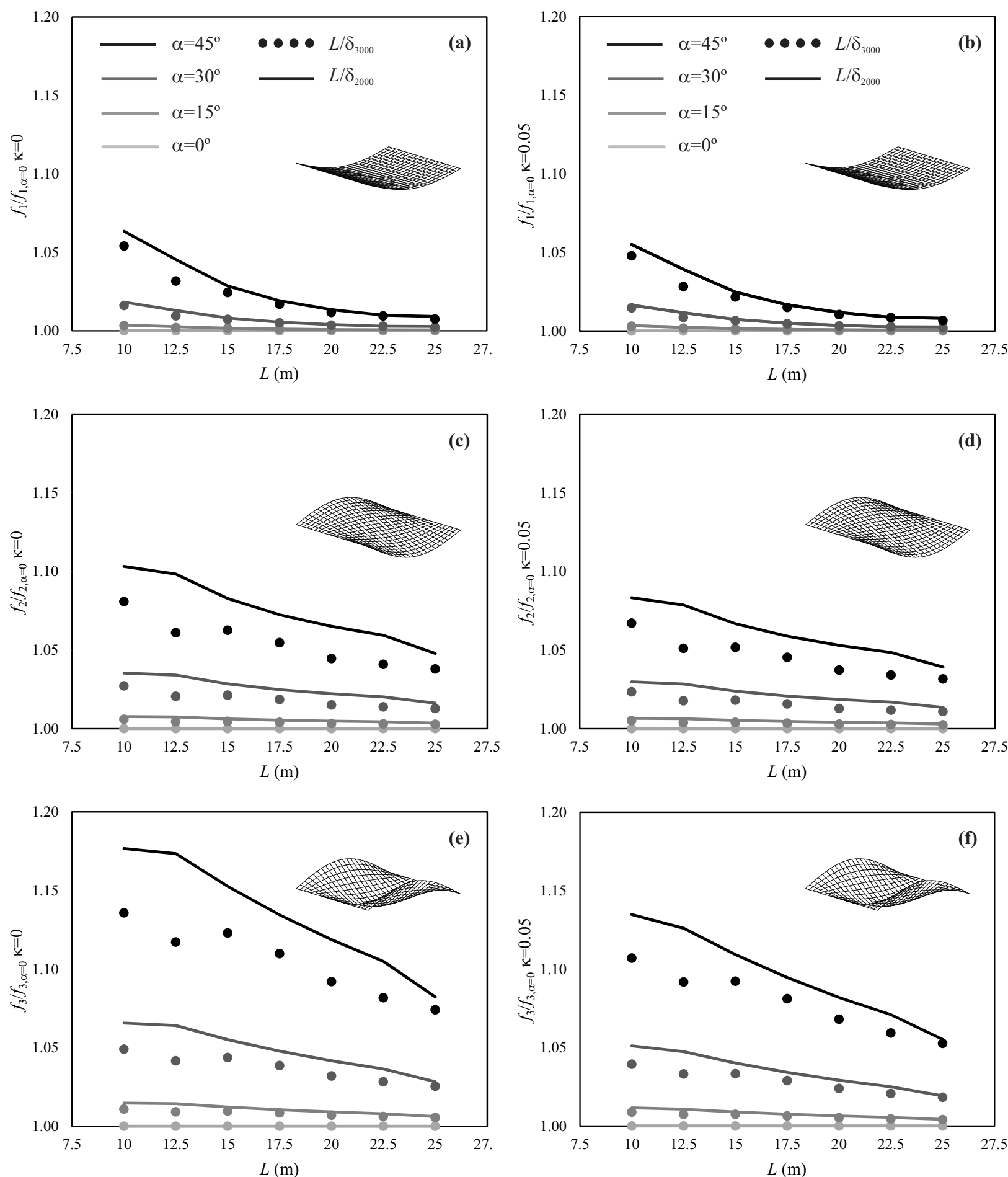

Figure 8: Relative variations of first three natural frequencies for the complete bridge catalogue with respect to the straight reference case $\left(\alpha=0^{\circ}\right)$. 
with respect to the theoretical predictions given in Table 1 are obtained as per

$$
E_{1 n}^{c 1}(\%)=\frac{K_{1 n}^{c 1}-K_{1 n, a n}^{c 1}}{K_{1 n, a n}^{c 1}} \cdot 100 \quad E_{1 n}^{\max , 2}(\%)=\frac{K_{1 n}^{\max , 2}-K_{1 n, a n}^{\max , 2}}{K_{1 n, a n}^{\max , 2}} \cdot 100
$$

where - an indicates the analytical values provided in Table 1 , and $K_{1 n}^{c 1}$ and $K_{1 n}^{\max , 2}$ are computed from the the orthotropic plate simply or elastically supported that are proposed in section 2 are good estimates in the case of oblique plates, even in highly skewed configurations. The previous conclusions are directly applicable to the $L / d_{k}$ theoretical ratios for maximized and cancelled resonances included in Table 2 , in virtue of Eqs. 13 and 14 . 

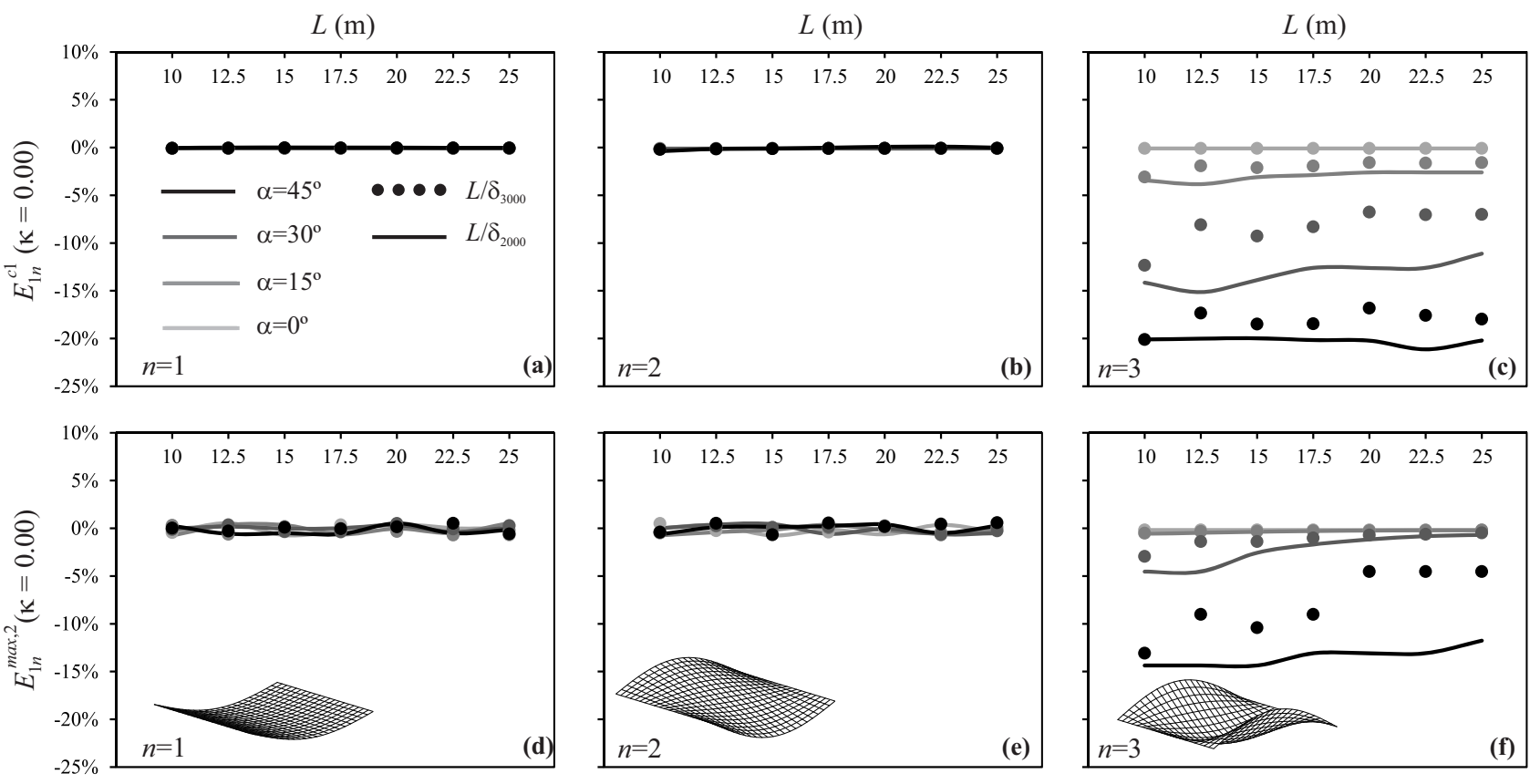

Figure 9: Variation of second maximum and first cancellation conditions in free vibration, $E_{1 m}^{\max , 2}(\%)$ and $E_{1 m}^{c 1}(\%)$, for the simply-supported bridges $\kappa=0.0$ of the bridge catalogue with respect to the straight reference case $\left(\kappa=0, \alpha=0^{\circ}\right)$.
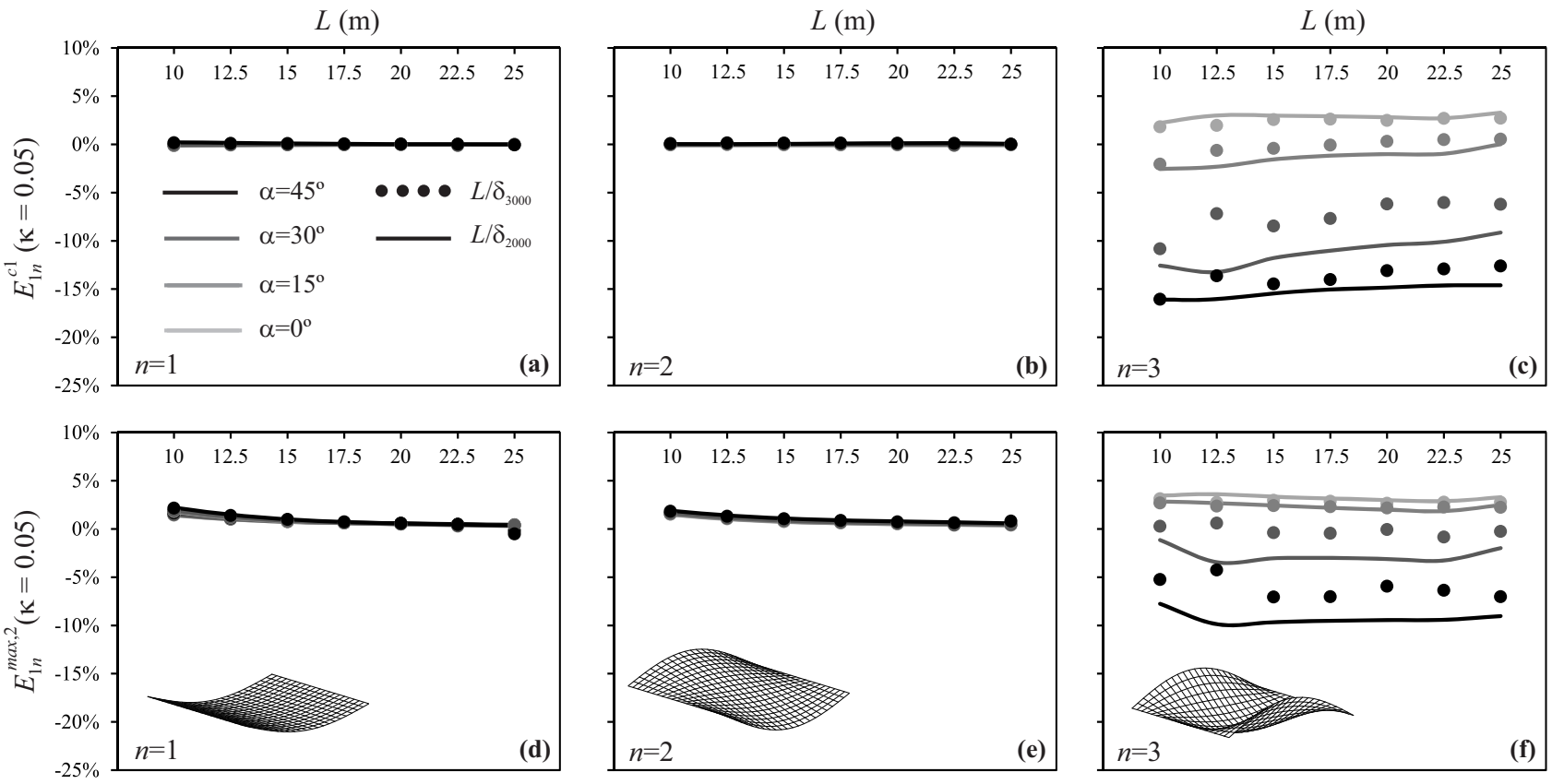

Figure 10: Variation of second maximum and first cancellation conditions in free vibration, $E_{1 m}^{\max , 2}(\%)$ and $E_{1 m}^{c 1}(\%)$, for the elastically-supported bridges $\kappa=0.05$ of the bridge catalogue with respect to the straight reference case $\left(\kappa=0.05, \alpha=0^{\circ}\right)$ 
bridge with $L=10 \mathrm{~m}, L / \delta_{2000}$ and $\alpha=0$ is selected as a representative example. Nevertheless the conclusions derived from it are applicable to the remaining cases. Fig. 11 shows the non-dimensional amplitude of the free vibrations for the second mode computed considering modal damping ratios $\zeta_{1 n}=[0,1,2,3] \%$. Fig. 11(a) corresponds to the SS case $(\kappa=0.0)$ and Fig. 11(b) to the ES case $(\kappa=0.05) . R_{1 n}$ is represented versus the speed parameter $K_{1 n}$ computed as per Eqs. 5 and 10 for $n=2$. The same evolution is identified for the first and third modes and only the case of the torsion mode is presented for the sake of brevity. The presence of damping has two main effects: (i) the modal amplitudes reduce, especially in the vicinity of the local maxima; (ii) the plate vibrations are not completely cancelled at the cancellation speeds. Nevertheless, and for the low levels of damping expected in railway bridges, cancellation phenomena will still be noticeable and the non-dimensional speeds for either cancellation or maximum free vibration should be adequately predicted with the theoretical values provided in Table 1.
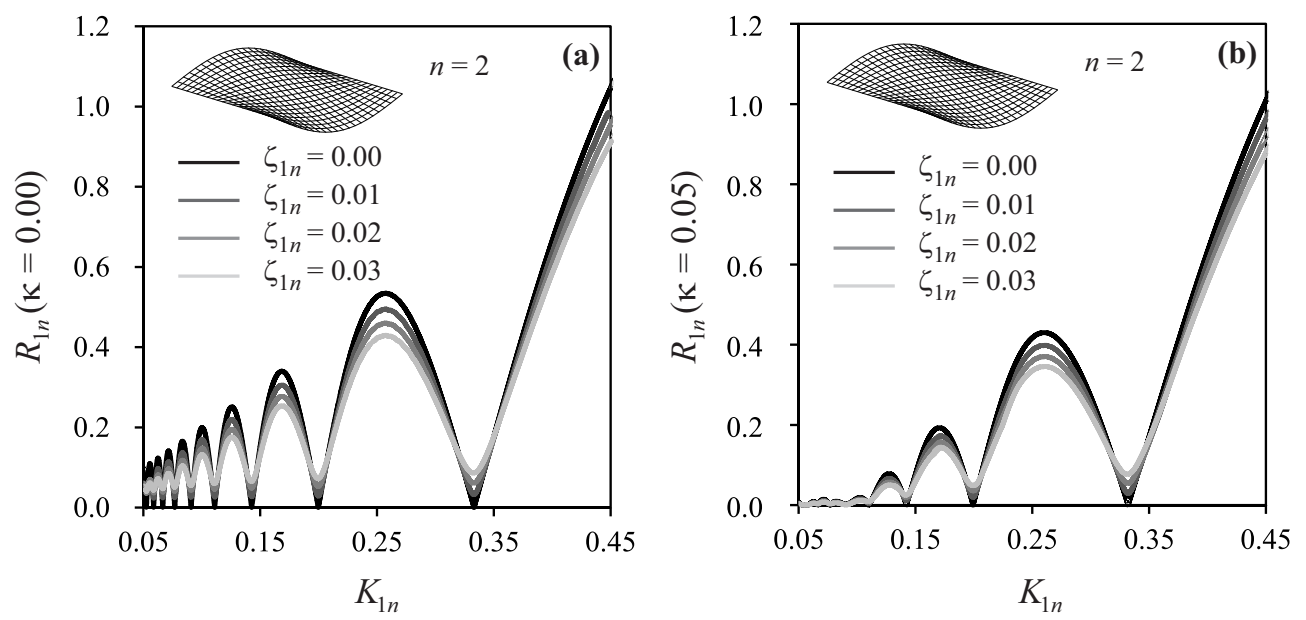

Figure 11: $R_{1 n}$ vs. $K_{1 n}$ for the second mode $(n=1 ; m=2) . L=10 \mathrm{~m}, L / \delta_{2000}, \alpha=0$. (a) $\kappa=0.0,(\mathrm{~b}) \kappa=0.05$.

In the next section the response of an existing bridge under HS railway traffic is presented. Two trains induce resonance of two different modes associated to the length of the passengers' cars $\left(d_{k}\right)$. The particular $L / d_{k}$ ratios are computed in each case and conclusions regarding their proximity to maximum and cancelled resonances analytical predictions, and the relation with the type of measured response are discussed.

\section{Experimental measurements on a real structure undergoing resonance}

\subsection{Structural description}

Arroyo Bracea bridge was specifically constructed in the early 1990s for the Madrid-Sevilla first HS railway line in Spain. It is composed by two identical SS bays with spans of $15.25 \mathrm{~m}$ and a width of $11.6 \mathrm{~m}$. The deck is made of a $25 \mathrm{~cm}$ thick concrete slab resting over five prestressed double-T girders with 


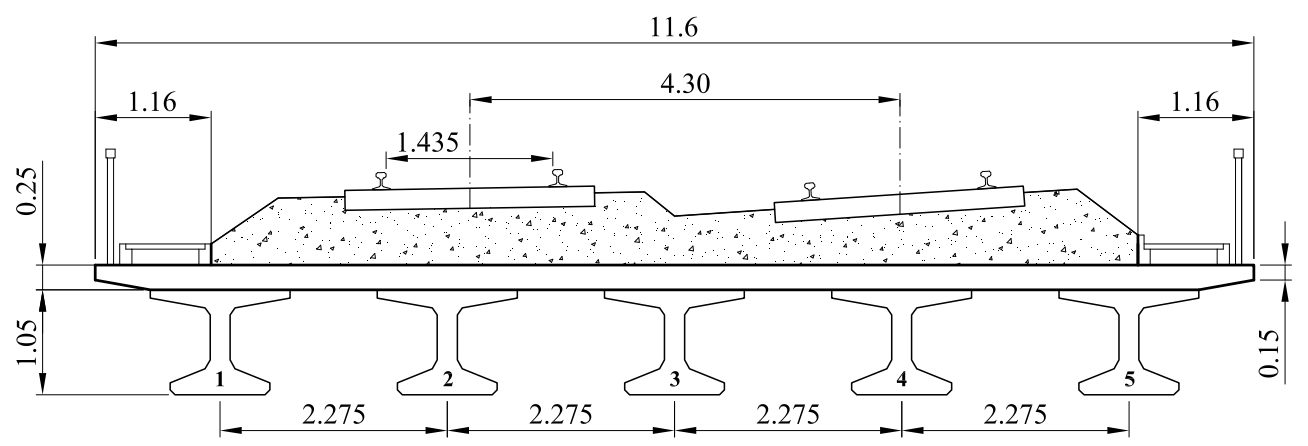

Figure 12: Arroyo Bracea bridge cross section.
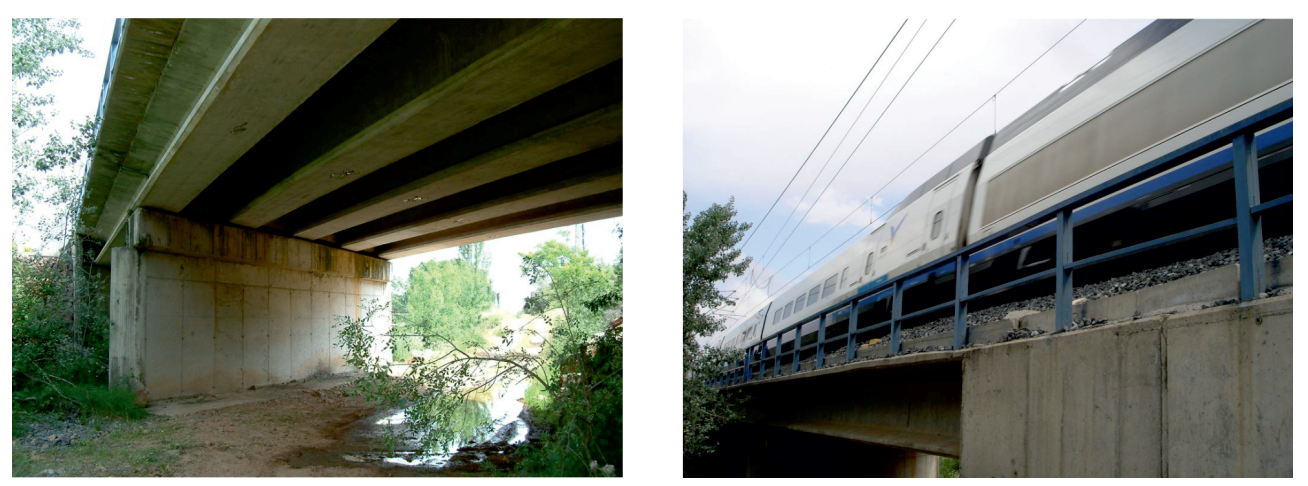

Figure 13: Arroyo Bracea bridge photographs.

$h_{g}=1.05 \mathrm{~m}$ (see Fig. 12). The girders lean on the supports through laminated rubber bearings. The vertical flexibility of the bearings in relation to the longitudinal bending stiffness of the deck $(L / \delta=3847)$ is such that $\kappa=0.1$. As per the substructure, the bridge deck is supported on reinforced concrete abutments in the outermost sections, and the inner sections of both bays lean on a pile foundation. The structure crosses the Bracea stream forming an angle of $45^{\circ}$ (see Fig. 13). The deck accommodates a double track ballasted platform. This structure is interesting from a dynamic point of view for the following reasons: (i) it presents five modes with natural frequencies below $30 \mathrm{~Hz}$; (ii) the flexibility of the supports and the obliquity of the deck are remarkable; (iii) due to the previous factors and the eccentricity of the excitation, the participation of modes different from the longitudinal bending ones (i.e. first torsion and first transverse bending modes) in the vertical acceleration response under railway loads is relevant. This bridge dynamic behaviour was numerically analysed by the authors in the past using an orthotropic plate FE model which showed a good correspondence between numerical predictions and experimental measurements, both under ambient vibration and under railway traffic. For a detailed description both of the structure and of the experimental campaign, briefly summarized in the next subsection, the reader is referred to [7]. 


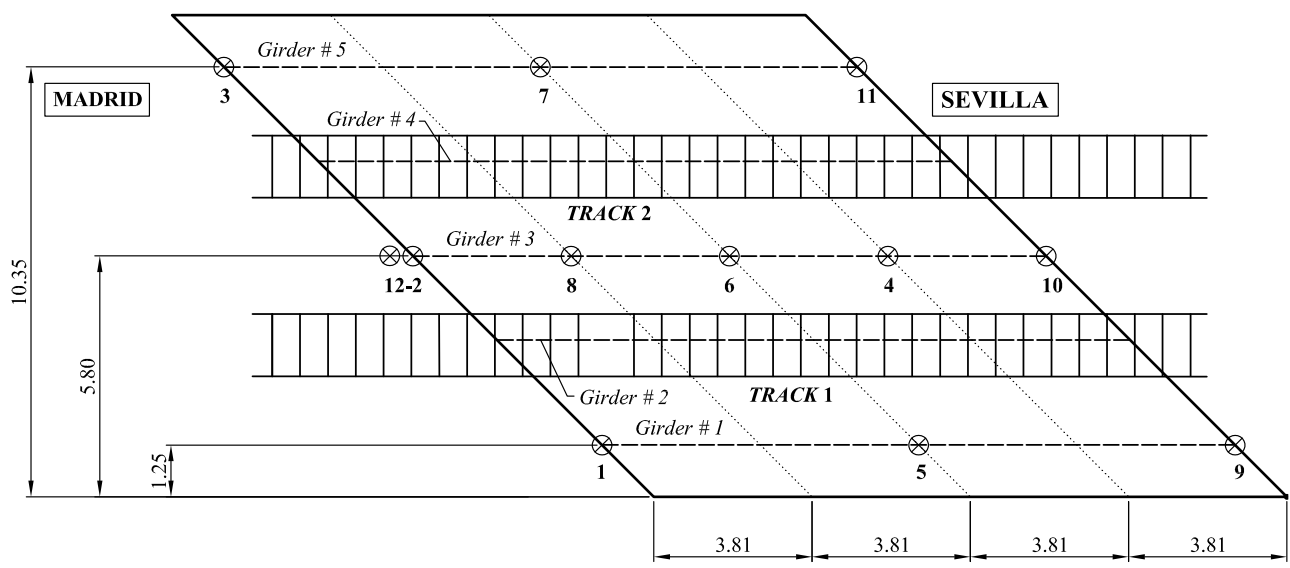

Figure 14: Arroyo Bracea bridge plan and sensors locations. bridge at $247 \mathrm{~km} / \mathrm{h}$. In Table 5 the theoretical resonant velocities associated to the first and second modes of the bridge deck (first longitudinal bending and first torsion modes) and to the length of the passengers' cars are included for the first three resonance orders. For the S103 and S130 trains these lengths are 24.8 and $13.1 \mathrm{~m}$, respectively.

In Table 5 two resonant speeds are highlighted: the third resonance of the fundamental mode induced by the S103 train and the second resonance of the first torsion mode induced by the S130 train. It can be 


\begin{tabular}{llllllll}
\hline Train & $V(\mathrm{~km} / \mathrm{h})$ & $d_{k}(\mathrm{~m})$ & $n$ & Mode & $V_{1 n}^{r 1}(\mathrm{~km} / \mathrm{h})$ & $V_{1 n}^{r 2}(\mathrm{~km} / \mathrm{h})$ & $V_{1 n}^{r 3}(\mathrm{~km} / \mathrm{h})$ \\
\hline S103 & 279 & 24.8 & 1 & $1^{\text {st }}$ L. bending & 825.0 & 412.5 & $\mathbf{2 7 5 . 0}$ \\
S130 & 247 & 13.1 & 2 & $1^{\text {st }}$ Torsion & 502.8 & $\mathbf{2 5 1 . 4}$ & 167.6 \\
\hline
\end{tabular}

Table 5: Arroyo Bracea bridge first theoretical resonance speeds associated to S103 and S130 passengers' cars lengths.

observed that these two theoretical critical velocities are in the vicinity of the actual circulating velocities of the trains and, therefore, could lead to a certain structural amplification.

In what follows the experimental structural response is shown for these two convoys. In Fig. 15 the bridge vertical acceleration is represented at sensor number 5 located at mid-span below girder \#1 for the two trains S103 and S130. Plots 15(a) and 15(b) show the acceleration time history and plots (c) and (d) the frequency content. From Fig. 15 it is evident that train S103 travelling at $279 \mathrm{~km} / \mathrm{h}$ induces resonance of the fundamental mode. The response progressively builds up and a peak in the frequency domain close to the identified fundamental frequency $(9.25 \mathrm{~Hz})$ clearly prevails. This type of response is consistent with that in the rest of the sensors. It should be stressed at this point that the maximum acceleration of the bridge is admissible despite the resonant situation according to the Serviceability Limit State of vertical acceleration in ballasted bridges $\left(a_{\max } \leq 3.5 \mathrm{~m} / \mathrm{s}^{2}\right)$. On the other hand, the circulation of S130 train at $247 \mathrm{~km} / \mathrm{h}$ induces a second resonance of the first torsion mode as well. The comparison of the bridge responses at sensors 5 and 6 (not included) confirms this statement. Also, in Fig. 15 it may be observed that the acceleration peak at the second mode frequency (torsion mode) of $10.63 \mathrm{~Hz}$ exceeds that of the fundamental mode.

It can be affirmed that both trains induce resonance on the bridge to a certain extent. Nevertheless, the amplification associated to the circulation of train S103 is much more evident, especially in the frequency domain, than the one associated to train S130, even though the former is a third resonance (one loaded and two empty cycles of oscillation between consecutive loads) and the latter is a second resonance (one loaded and one empty cycle of oscillation between consecutive loads). In the view of the authors this difference in amplitude could be related, at least partially, with the level of free vibrations that every axle load leaves on the structure at the particular velocity of circulation in each mode. If ratios $L / d_{k}$ are calculated for both trains:

$$
\begin{aligned}
& \left(\frac{L}{d_{S 103}}\right)=\frac{15.25}{24.8}=0.61 \simeq\left(\frac{L}{d_{k}}\right)_{m=1, j=3}^{m a x, i=2}=\left.0.6131\right|_{\kappa=0.1} \\
& \left(\frac{L}{d_{S 130}}\right)=\frac{15.25}{13.1}=1.16 \simeq\left(\frac{L}{d_{k}}\right)_{m=1, j=2}^{c, i=2}=\left.1.1810\right|_{\kappa=0.1}
\end{aligned}
$$

Eq. 16a shows how the $L / d_{k}$ ratio for the S103 train is close to a condition of maximum resonance for the third resonance of modes with $m=1$ (see Table 2). On the other hand, Eq. $16 \mathrm{~b}$ shows that the $L / d_{k}$ ratio for the S130 train is close to a cancellation condition for second resonances of also modes with $\mathrm{m}=1$. 

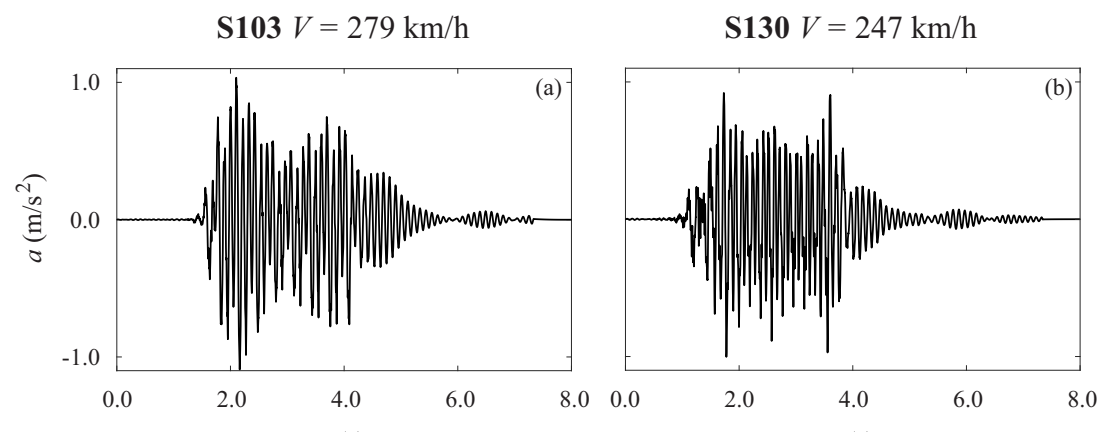

$t(\mathrm{~s})$
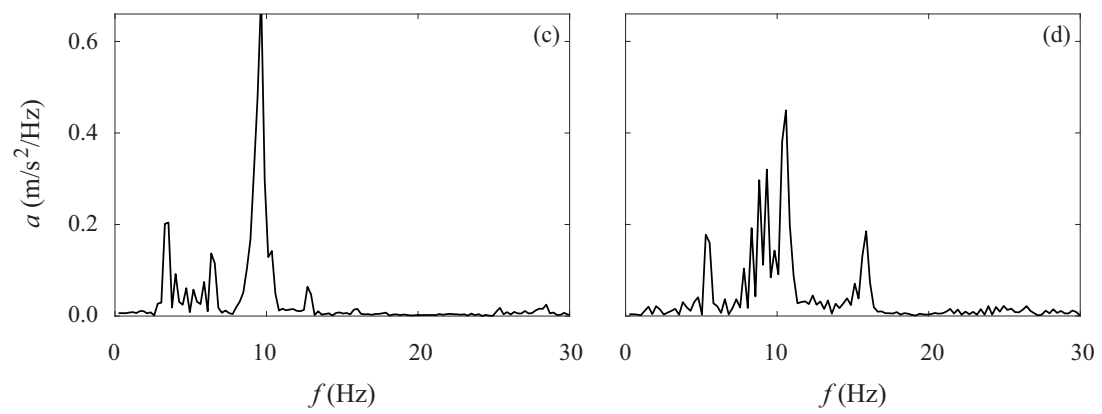

Figure 15: Vertical acceleration measured in Arroyo Bracea bridge under S103 and S130 trains.

The amplitude of the free vibrations associated to a particular speed, and the effect of it on the character of a resonant response is evidently a basic phenomenon. Other factors will affect the resonant response of the bridge such as those related to vehicle-track-structure interaction or soil-structure interaction. Nevertheless, being able to predict the basic geometrical effects of the loads at resonance will permit a much better understanding of more complex coupling mechanisms in the vibrational response of railway bridges.

\section{Conclusions}

The free vibration response of simply-supported and elastically-supported orthotropic rectangular plates traversed by a single load moving at constant speed is formulated in this work. Cancellation and maximum free vibration conditions alternatively take place as the speed of the load increases. Analytical non-dimensional speeds associated to these situations are proposed for a generic straight plate with simple or elastic supports.

When the plate is traversed by a sequence of equidistant loads travelling at constant speed parallel to the plate free edges resonance may be induced on the former. The main application of this study is the dynamic response of railway bridges with simply-supported orthotropic decks, due to its relation with the Serviceability Limit State of vertical acceleration, which is nowadays one of the most strict requirements for structures located in High-Speed lines. When a resonant speed coincides or is close to a maximum free 
vibration condition, a prominent resonant peak in the response of the deck, should be expected. On the other hand, when it is close to a cancellation condition, the resonant response will be mild or may not even be perceptible for the particular mode. By equating maximum free vibration and cancellation conditions to the resonance ones, bridge length-to-characteristic distance ratios are obtained and presented for maximum or cancelled resonances for SS and ES rectangular orthotropic plates. Why these conditions coincide with those of a simple beam, in the SS case, and are well approximated by those of an ES beam, in the ES case is justified through the analytical formulation.

The previous analytical conditions, obtained in the ideal (undamped and straight) case, are presented in simple tables and are proposed for any bridge deck with an orthotropic plate behaviour. Their adequacy in order to predict the real values of cancellation and maximum free vibration velocities, or the corresponding $L / d_{k}$ ratios for maximum resonance or cancellation of it in the case of oblique decks, with different longitudinal bending stiffness and flexibility of the supports, is investigated numerically. To this end a comprehensive bridge catalogue of 112 skewed bridges covering realistic parameters is pre-designed and a sensitivity study is performed. The main conclusions extracted are:

- For the $1^{\text {st }}$ longitudinal bending and $1^{\text {st }}$ torsion modes, the non-dimensional values for the speeds causing cancellation or maximum free vibration of the orthotropic plate that are proposed in section 2 are very good estimates for the real values in the case of oblique plates, even in highly skewed configurations, both in the simply-supported and in the elastically supported case.

- The first transverse bending mode, generally the third in frequency order in the bridge typologies under study, is more affected by the plate obliquity and incurred errors approach $20 \%$ for the highest obliquity levels and most flexible bridges considered.

- In the presence of damping the modal amplitudes reduce, specially in the vicinity of the local maxima, and the plate vibrations are not completely cancelled at the cancellation speeds. Nevertheless, and for the low levels of damping expected in railway bridges, cancellation phenomena will still be noticeable and the non-dimensional speeds for either cancellation or maximum free vibration should be adequately predicted with the analytical values provided.

Finally the experimental response of an existing bridge under HS railway traffic is presented. Two trains induce resonance of two different modes associated to the length of the passengers' cars. The level of free vibrations is evaluated and discussed in relation to the type of response in each case.

The amplitude of the free vibrations associated to a particular speed, and the effect of it on the character of a resonant response is evidently a basic phenomenon. Other factors will affect the resonant response of the bridge such as those related to vehicle-track-structure interaction or soil-structure interaction. Nevertheless, being able to predict the basic geometrical effects of the loads at resonance will permit a much better 
understanding of more complex coupling mechanisms in the vibrational response of railway bridges.

\section{Acknowledgements}

The authors would like to acknowledge the financial support provided by (i) the Spanish Ministries of Economy and Competitiveness and of Education under research project [BIA2016-75042-C2] and under "José Castillejo" grant CAS18/00080 for mobility of researchers; (ii) the Andalusian Scientific Computing Centre (CICA); (iii) Generalitat Valenciana AICO/2019/175 research project for consolidable groups.

\section{Appendix A.}

In Eq. 2:

$$
\begin{gathered}
Y_{i j}(y)=\frac{\delta_{i j} \cosh \left(\phi_{i j} y / L\right)+\gamma_{i j} \cos \left(\vartheta_{i j} y / L\right)}{\gamma_{i j} \delta_{i j}\left(\cosh \left(\phi_{i j} B / L\right)-\cos \left(\vartheta_{i j} B / L\right)\right)}-\frac{\vartheta_{i j} \gamma_{i j} \sinh \left(\phi_{i j} y / L\right)+\phi_{i j} \delta_{i j} \sin \left(\vartheta_{i j} y / L\right)}{\vartheta_{i j} \gamma_{i j}^{2}\left(\sinh \left(\phi_{i j} B / L\right)-\phi_{i j} \delta_{i j}^{2} \sin \left(\vartheta_{i j} B / L\right)\right)} \\
\phi_{i j}=\frac{i \pi}{\sqrt{D_{y}}}\left[\left(H^{2}-D_{x} D_{y}+\frac{\rho h D_{y} L^{4} \omega_{i j}^{2}}{i^{4} \pi^{4}}\right)^{\frac{1}{2}}+H\right]^{\frac{1}{2}} \\
\vartheta_{i j}=\frac{i \pi}{\sqrt{D_{y}}}\left[\left(H^{2}-D_{x} D_{y}+\frac{\rho h D_{y} L^{4} \omega_{i j}^{2}}{i^{4} \pi^{4}}\right)^{\frac{1}{2}}-H\right]^{\frac{1}{2}} \\
\delta_{i j}=D_{y} \vartheta_{i j}^{2}+i^{2} \pi^{2} \frac{D_{1}+D_{2}}{2} \\
\gamma_{i j}=D_{y} \phi_{i j}^{2}+i^{2} \pi^{2} \frac{D_{1}+D_{2}}{2}
\end{gathered}
$$

In Eqs. A.1b and A.1c $\omega_{i j}$ is the circular frequency of the orthotropic plate related to the roots of the frequency equation (Eq. A.2b) $\omega_{i j}$ as per

$$
\begin{gathered}
\omega_{i j}=\frac{\sqrt{D_{y} \phi_{i j}^{4}-2 i^{2} \pi^{2} H \phi_{i j}^{2}+i^{4} \pi^{4} D_{x}}}{L^{2} \sqrt{\rho h}} \\
\left(\vartheta_{i j}^{2} \gamma_{i j}^{4}-\phi_{i j}^{2} \delta_{i j}^{4}\right) \sinh \left(\phi_{i j} \frac{B}{L}\right) \sin \left(\vartheta_{i j} \frac{B}{L}\right)+2 \phi_{i j} \vartheta_{i j} \gamma_{i j}^{2} \delta_{i j}^{2}\left(\cosh \left(\phi_{i j} \frac{B}{L}\right) \cos \left(\vartheta_{i j} \frac{B}{L}\right)-1\right)=0
\end{gathered}
$$

In Eq,8, corresponding to the transverse displacement of a plate elastically-supported along $X$ and free-free (FF) along $Y$, the functions $X_{i}(x)$ and $Y_{j}(y)$ may be expressed as [33, 34,

$$
\begin{array}{r}
X_{i}(x)=\sin \left(\frac{\lambda_{i} x}{L}\right)+\sinh \left(\frac{\lambda_{i} x}{L}\right) \frac{\sin \left(\lambda_{i}\right)}{\sinh \left(\lambda_{i}\right)}+\gamma_{1 i}\left(\cos \left(\frac{\lambda_{i} x}{L}\right)+\cosh \left(\frac{\lambda_{i} x}{L}\right)+\gamma_{2 i} \sinh \left(\frac{\lambda_{i} x}{L}\right)\right) \\
\gamma_{1 i}=\frac{\sinh \left(\lambda_{i}\right)-\sin \left(\lambda_{i}\right)}{(2 / \kappa)\left(\pi / \lambda_{n}\right)^{3} \sinh \left(\lambda_{i}\right)+\cos \left(\lambda_{i}\right)-\cosh \left(\lambda_{i}\right)}, \quad \gamma_{2 i}=\frac{\cos \left(\lambda_{i}\right)-\cosh \left(\lambda_{i}\right)}{\sinh \left(\lambda_{i}\right)} \\
Y_{j}(y)=\left(\cos \left(\frac{\lambda_{j} y}{B}\right)+\cosh \left(\frac{\lambda_{j} y}{B}\right)\right)-\frac{\cos \left(\lambda_{j}\right)-\cosh \left(\lambda_{j}\right)}{\sin \left(\lambda_{j}\right)-\sinh \left(\lambda_{j}\right)}\left(\sin \left(\frac{\lambda_{j} y}{B}\right)+\sinh \left(\frac{\lambda_{j} y}{B}\right)\right)
\end{array}
$$


where parameters $\lambda_{i}$ and $\lambda_{j}$ in the two directions are obtained from the respective frequency equations of the ES (Eq. A.5a) and the FF (Eq. A.5b) beams as per

$$
\begin{aligned}
\left(\frac{\pi^{3}}{\kappa}\right)^{2}+\frac{\pi^{3}}{\kappa} \lambda_{i}^{3} \frac{\sinh \left(\lambda_{i}\right) \cos \left(\lambda_{i}\right)-\cosh \left(\lambda_{i}\right) \sin \left(\lambda_{i}\right)}{\sin \left(\lambda_{i}\right) \sinh \left(\lambda_{i}\right)}+\lambda_{i}^{6} \frac{1-\cos \left(\lambda_{i}\right) \cosh \left(\lambda_{i}\right)}{2 \sin \left(\lambda_{i}\right) \sinh \left(\lambda_{i}\right)}=0 \\
\cos \left(\lambda_{j}\right) \cosh \left(\lambda_{j}\right)-1=0
\end{aligned}
$$

Note that Eq. A.5b leads to two rigid body modes corresponding to $\lambda_{j}=0$. Then, the circular frequency of the $i j$-th mode of the ES plate can be obtained by substitution of Eqs. A.3. A.5 into Eq. A.6

$$
\begin{array}{r}
\bar{\omega}_{i j}^{2}=\frac{1}{M_{m n}} \int_{0}^{L} \int_{0}^{B}\left(D_{x} \frac{\partial^{4} \Phi_{i j}}{\partial x^{4}}+2 H \frac{\partial^{4} \Phi_{i j}}{\partial x^{2} \partial y^{2}}+D_{y} \frac{\partial^{4} \Phi_{i j}}{\partial y^{4}}\right) \Phi_{i j}(x, y) d y d x \\
M_{m n}=\int_{0}^{L} \int_{0}^{B} \rho h \Phi_{i j}(x, y)^{2} d y d x
\end{array}
$$

\section{References}

[1] ADIF, Railway network statement 2019 (in Spanish) Tech. rep., Ministerio de Fomento - Gobierno de España (2019). URL http://www.adifaltavelocidad.es/

[2] ERRI D214, Rail bridges for speeds $>200 \mathrm{~km} / \mathrm{h}$. Final report. Part a. Synthesis of the results of D 214 research, European Rail Research Institute, 1999.

[3] L. Frýba, Dynamic behaviour of bridges due to high-speed trains, in: Workshop bridges for high-speed railways, Porto, 2004, pp. 137-158.

[4] W. Hoorpah, Dynamic calculations of high-speed railway bridges in France some case studies, in: T. \&. Francis (Ed.), Dynamics of High-Speed Railway Bridges, no. 978-0-203-89540-5, 2008, pp. 133-146. doi:https://doi.org/10.1201/ 9780203895405

[5] M. Zacher, M. Baebler, Dynamic behaviour of ballast on railway bridges, in: T. Francis (Ed.), Dynamics of High-Speed Railway Bridges, no. 978-0-203-89540-5, 2008, pp. 99-112. doi:https://doi.org/10.1201/9780203895405

[6] I. Bisús, Tipología de viaductos en las líneas de alta velocidad en España, Master's thesis, E.T.S. Ingenieros de Caminos, Canales y Puertos de Barcelona, Universitat Politecnica de Catalunya (2009).

URL http://hdl.handle.net/2099.1/12582

[7] P. Galvín, A. Romero, E. Moliner, M. Martínez-Rodrigo, Two FE models to analyse the dynamic response of short span simply-supported oblique high-speed railway bridges: Comparison and experimental validation, Engineering Structures 167 (2018) 48-64. doi:https://doi.org/10.1016/j.engstruct.2018.03.052

[8] Y. Yang, J. Yau, L. Hsu, Vibration of simple beams due to trains moving at high speeds, Engineering Structures 19(11) (1997) 936-944.

[9] E. Savin, Dynamic amplification factor and response spectrum for the evaluation of vibrations of beams under successive moving loads, Journal of Sound and Vibration 248 (2) (2001) $267-288$.

[10] P. Museros, E. Moliner, M. Martínez-Rodrigo, Free vibrations of simply-supported beam bridges under moving loads: Maximum resonance, cancellation and resonant vertical acceleration, Journal of Sound and Vibration 332 (2) (2013) 326 -345 .

[11] M. Simsek, M. Aydn, H. H. Yurtcu, J. N. Reddy, Size-dependent vibration of a microplate under the action of a moving load based on the modified coupled stress theory, Acta Mechanica 226 (2015) 38073822. doi:10.1007/s00707-015-1437-9

[12] J. Gbadeyan, O. S.T., Dynamic response of a mindlin elastic rectangular plate under a distributed moving mass, Journal of Sound and Vibration 182 (5) (1995) 677-695. doi:10.1006/jsvi.1995.0226 
[13] M. Shadnam, M. Mofid, J. Akin, On the dynamic response of rectangular plate, with moving mass, Thin-walled Structures 39 (2001) 797806. doi:10.1016/S0263-8231(01)00025-8

[14] S. Lee, S. Yhim, Dynamic analysis of composite plates subjected to multi-moving loads based on a third order theory, International Journal of Solids and Structures 41 (2004) 44574472. doi:10.1016/j.ijsolstr.2004.03.021

[15] F. Au, M. Wang, Sound radiation from forced vibration of rectangular orthotropic plates under moving loads, Journal of Sound and Vibration 281 (2005) 10571075. doi:10.1016/j.jsv.2004.02.005

[16] J. Gbadeyan, M. Dada, Dynamic response of a mindlin elastic rectangular plate under a distributed moving mass, International Journal of Mechanical Sciences 48 (2006) 323340. doi:10.1016/j.ijmecsci.2005.09.005

[17] P. Malekzadeh, A. Fiouz, H. Razi, Three-dimensional dynamic analysis of laminated composite plates subjected to moving load, Composite Structures 90 (2) (2009) 105-114. doi:10.1016/j.compstruct.2009.02.008

[18] P. Malekzadeh, M. Haghighi, M. Gholami, Dynamic response of thick laminated annular sector plates subjected to moving load, Composite Structures 92 (2010) 155163. doi:10.1016/j.compstruct.2009.07.020

[19] E. Ghafoori, M. Asghari, Dynamic analysis of laminated composite plates traversed by a moving mass based on a first-order theory, Composite Structuctures 92 (2010) 18651876. doi:10.1016/j.compstruct.2010.01.011

[20] X. Zhu, S. Law, Dynamic behavior of orthotropic rectangular plates under moving loads, Journal of Engineering Mechanics 129(1) (2003) 79-87.

[21] A. Nikkhoo, M. Ebrahimzadeh, S. Azam, J. Amiri, Vibration of a thin rectangular plate subjected to series of moving inertial loads, Mechanics research communications 55 (2014) 105-113. doi:10.1016/j.mechrescom.2013.10.009

[22] M. Ebrahimzadeh, N. Attari, A. Nikkhoo, S. Mariani, Resonance of a rectangular plate influenced by sequential moving masses, Coupled Systems Mechanics 5 (1) (2016) 87-100. doi:10.12989/csm.2016.5.1.087

[23] M. Martínez-Rodrigo, P. Museros, Optimal design of passive viscous dampers for controlling the resonant response of

orthotropic plates under high-speed moving loads, Journal of Sound and Vibration 330 (2011) 13281351. doi:10.1016/j. jsv.2010.10.017

[24] E. Moliner, M. Martínez-Rodrigo, P. Museros, Dynamic performance of existing double track railway bridges at resonance with the increase of the operational line speed, Engineering Structures 132 (2017) 98 - 109. doi:http://dx.doi.org/10. $1016 / j$.engstruct.2016.11.031 URL http://www.sciencedirect.com/science/article/pii/S0141029616312639

[25] W. H. N.J. Huffington, On the transverse vibrations of rectangular orthotropic plates, Journal of Applied Mechanics 25 (1958) $389-395$.

[26] A. Leissa, Vibration of plates, Tech. Rep. NASA SP-160, NASA (1969).

[27] CEN, EN 1991-2, Eurocode 1: Actions on Structures - Part 2: Traffic loads on bridges, European Committee for Standardization, Brussels, 2002.

[28] E. Moliner, A. Romero, P. Galvín, M. Martínez-Rodrigo, Effect of the end cross beams on the railway induced vibrations of short girder bridges, Engineering Structures 201 (109728) (2019) 1-16.

[29] C. A. Felippa, Refined finite element analysis of linear and nonlinear two dimensional structures, Ph.D. thesis, University of California, Berkeley (1966).

[30] M. Martínez-Rodrigo, J. Lavado, P. Museros, Dynamic performance of existing high-speed railway bridges under resonant

․ conditions retrofitted with fluid viscous dampers Engineering Structures 32 (3) (2010) 808 - 828. doi:https://doi.org/ $10.1016 / j$.engstruct.2009.12.008 URL http://www.sciencedirect.com/science/article/pii/S0141029609003927

[31] A. R. Cusens, R. P. Pama, Bridge deck analysis, John Wiley \& Sons, 1975.

[32] E. Reynders, System identification methods for (operational) modal analysis: review and comparison, Archives of Computational Methods in Engineering 19 (1) (2010) 51-124. 
[33] I. Karnovsky, O. Lebed, Formulas for structural dynamics, McGraw Hill, 2000.

$475[34]$ S. Rao, Vibration of continuous systems, John Wiley \& Sons, 2007. 